\title{
Streamflow and Water-Quality Data for Bear Butte Creek Downstream of Sturgis, South Dakota, 1998-2000
}

By Joyce E. Williamson

Open-File Report 00-430

Prepared in cooperation with the South Dakota Department of Environment and Natural Resources 


\section{U.S. Department of the Interior}

Bruce Babbitt, Secretary

\section{U.S. Geological Survey}

Charles G. Groat, Director

The use of firm, trade, and brand names in this report is for identification purposes only and does not constitute endorsement by the U.S. Geological Survey.

Rapid City, South Dakota: 2000

For additional information write to:

\section{District Chief}

U.S. Geological Survey

1608 Mt. View Road

Rapid City, SD 57702

Copies of this report can be purchased from:

U.S. Geological Survey

Information Services

Box 25286

Denver, CO 80225-0286 


\section{CONTENTS}

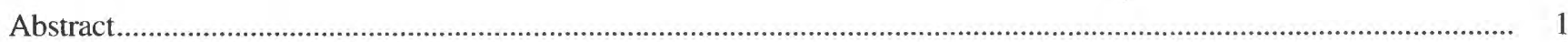

Introduction

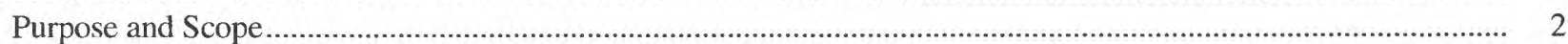

Description of Bear Butte Creek Basin ...............................................................................................

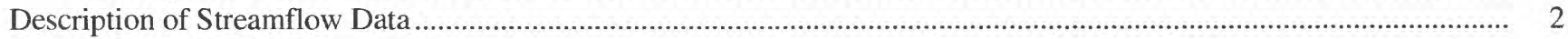

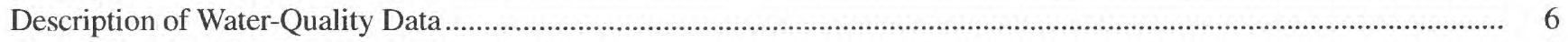

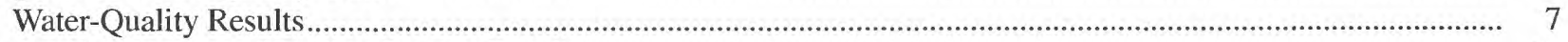

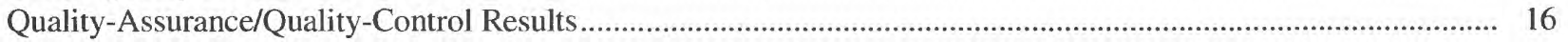

Beneficial-Use Designations and Water-Quality Criteria..................................................................... 17

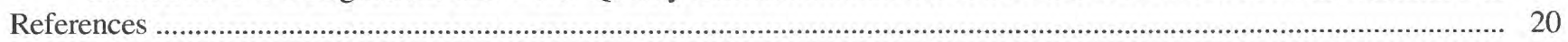

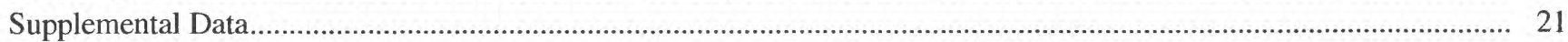

\section{FIGURES}

1. Map showing the location of Bear Butte Creek Basin.

2. Map showing the locations of selected gaging stations and water-quality sampling sites ..................................... 3

3. Graphs showing miscellaneous discharge measurements for selected streamflow-gaging stations, May 1998 to May 2000.

4. Continuous stage recordings, stage at miscellaneous measurements, and storm-event stage at Bear Butte Creek at Sturgis

5. Graph showing comparison of miscellaneous discharge measurements during water years 1998-2000 to hydrographs of long-term statistical range of flows (1945-72) at Bear Butte Creek near Sturgis ...

6. Boxplots by site for selected constituents for Bear Butte Creek from June 1998 to May 2000.

7. Trilinear diagrams showing proportional concentrations of major ions for select sites

8. Graphs showing relation between water hardness and freshwater aquatic-life standards for acute and chronic toxicity of selected trace elements

\section{6}

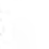

16.




\title{
Streamflow and Water-Quality Data for Bear Butte Creek Downstream of Sturgis, South Dakota, 1998-2000
}

\author{
By Joyce E. Williamson
}

\section{ABSTRACT}

This report presents miscellaneous streamflow and water-quality data that were collected from June 1998 through May 2000 for the Bear Butte Creek Water Quality Study. This study was completed in cooperation with the South Dakota Department of Environment and Natural Resources with the objective of collecting baseline data along the lower reach of Bear Butte Creek.

Miscellaneous discharge and stage data are presented for four sites along Bear Butte Creek-one immediately below the City of Sturgis, one below inflows from Cottle Creek, another below inflows from Spring Creek, and the last just upstream from the confluence with the Belle Fourche River. Continuous stage is presented for the first site. Discharge was measured at all sites during monthly site visits as well as during four storm events.

Results from water-quality data analyzed by the South Dakota State Health Laboratory, as well as results from quality assurance/quality control samples, are presented. Samples were collected at the same time that discharge measurements were made. Beneficial-use designations and waterquality criteria for selected segments of Bear Butte Creek are presented.

\section{INTRODUCTION}

The City of Sturgis operates a municipal wastewater lagoon facility adjacent to Bear Butte Creek.
Treated effluent from the facility occasionally is used for irrigation purposes on nearby land. In 1996, with significantly higher inflows, limited evaporation, and no need for the treated water for irrigation purposes, the lagoons were full and in order to protect the lagoon system, the City of Sturgis discharged treated water to Bear Butte Creek. Following the discharge, the South Dakota Department of Environment and Natural Resources (DENR) required the City of Sturgis to develop a plan to ensure that a similar situation did not reoccur. One of the options that was considered by the City was to apply for a discharge permit. DENR found that very little water-quality data existed for Bear Butte Creek near and below the City of Sturgis from which discharge limits could be based. In addition to this, Bear Butte Creek was included on the State 303D list for establishment of total maximum daily loads (TMDL). DENR approached the U.S. Geological Survey (USGS) to assist in streamflow characterization and the collection of water-quality samples.

The general objectives of the study were to: (1) establish one continuous and three miscellaneous streamflow-gaging sites on Bear Butte Creek; and (2) determine existing baseline water-quality conditions in Bear Butte Creek so that the State can begin to determine temporal variations in streamflow and variations in water quality in relation to streamflow quantity.

Data collection and analysis completed for the study provide the information needed by DENR to begin to assess the hydrologic conditions in this area. The information obtained will provide data for developing technically based TMDL's within a basin where only limited streamflow and water-quality data previously existed. 


\section{Purpose and Scope}

This report presents selected discharge and water-quality data collected in the Bear Butte Creek Basin downstream from the City of Sturgis. Miscellaneous measurements from four gaging stations are presented. Continuous discharge is published in the annual Water Resources Data Report for South Dakota (U.S. Geological Survey, 1999, 2000). Results from water-quality samples analyzed by the South Dakota State Health Laboratory (SHL) are included herein, as well as the quality-assurance/quality-control samples (QA/QC) analyzed by the SHL and the USGS National Water Quality Laboratory (NWQL). Hydrographs. scatter plots, boxplots, and trilinear diagrams are used for comparing changes in flow and concentration levels within the basin.

\section{Description of Bear Butte Creek Basin}

Bear Butte Creek originates south of Deadwood, South Dakota, and drains the area between Whitewood Creek on the north and Elk Creek and Alkali Creek on the south (fig. 1). Tributaries in the upper reach include Strawberry Creek, Park Creek, Two Bit Creek, and Boulder Creek. For most months of the year, Bear Butte Creek loses all or most of its flow as it crosses the Madison Limestone and Minnelusa Formation outcrops. The streambed generally is dry from about 3 miles west of Sturgis to the east edge of Sturgis. Springs and well discharges generally maintain some perennial flow in Bear Butte Creek for the area near the eastern edge of Sturgis to upstream of the Sturgis wastewater lagoon facility. Cottle Creek and Spring Creek provide tributary inflows within the lower reach of Bear Butte Creek.

The upper part of Bear Butte Creek is representative of a mountain-type stream with a rock/cobble bottom and higher gradients. Historical mining, primarily for gold, in this part of the basin has impacted stream quality. This is evident on Strawberry Creek below the Gilt Edge mine, which currently is proposed as a superfund site. Bear Butte Creek below Sturgis is more representative of a prairie-type stream with meanders and a mud bottom. Land use adjacent to the stream and tributaries is predominantly agricultural. Sturgis and close-by housing developments probably provide some urban land-use influence.

\section{DESCRIPTION OF STREAMFLOW DATA}

This section presents the miscellaneous discharge measurements collected along Bear Butte Creek downstream of the City of Sturgis. Four sites were established within the basin from immediately upstream from the City's Wastewater Treatment Facility to just above the confluence with the Belle Fourche River. Table 1 presents site information, and figure 2 displays the location of the sites within the basin. For simplification, sites may be referenced by a site identification number (site 1 , site 2 , etc.) within this text and on related figures. Site 2, Bear Butte Creek below Sturgis, and site 3, Bear Butte Creek near Sturgis, were located immediately downstream from inflows from Cottle Creek and Spring Creek, respectively. Cottle Creek drains the area south of Bear Butte Creek and Spring Creek drains an area north of Bear Butte Creek.

Miscellaneous discharge measurements were obtained monthly at each of the four sites and during storm events (table 2, fig. 3). Flows generally increased between site 1 and site 2 (Cottle Creek inflows) and between site 2 and site 3 (Spring Creek inflows). With the exception of storm events, flow between site 3 and site 4 did not vary considerably. No flow occurred at site 4 in September 1998. During the first storm event (June 18, 1998), flows decreased from site 1 to site 2 , which probably resulted from attenuation in filling the stream channel and alluvial storage, and then increased from site 2 to site 3 and from site 3 to site 4.

Table 1. Site information for selected streamflow-gaging stations and water-quality sampling sites

\begin{tabular}{lcclccc}
\hline \multicolumn{1}{c}{ Site number } & $\begin{array}{c}\text { Site } \\
\text { identification }\end{array}$ & \multicolumn{1}{c}{ Site name } & $\begin{array}{c}\text { Latitude } \\
\text { Longitude }\end{array}$ & $\begin{array}{c}\text { Elevation } \\
\text { (feet) }\end{array}$ \\
\hline 06437400 & 1 & Bear Butte Creek at Sturgis, SD & 442444 & 1032910 & 3.320 .00 \\
442811103205000 & 2 & Bear Butte Creek below Sturgis, SD & 442811 & 1032050 & $2,930.00$ \\
06437500 & 3 & Bear Butte Creek near Sturgis, SD & 442853 & 1031631 & $2,779.91$ \\
443048103091400 & 4 & Bear Butte Creek near mouth, near Vale, SD & 443048 & 1030914 & $2,590.00$ \\
\hline
\end{tabular}




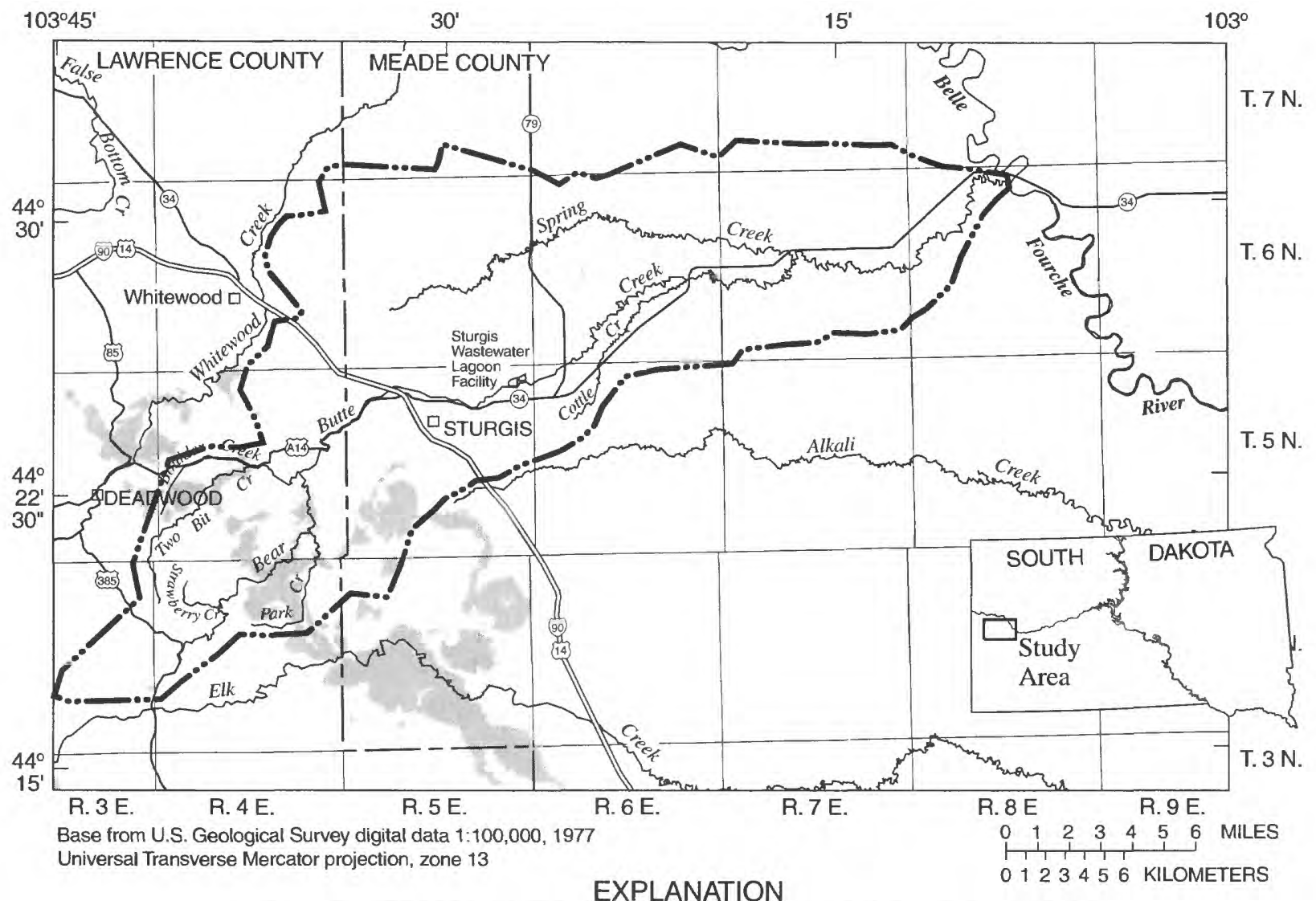

OUTCROP OF MADISON LIMESTONE (from Strobel and others, 1999)

GENERALIZED BASIN BOUNDARY

Figure 1. Location of Bear Butte Creek Basin.

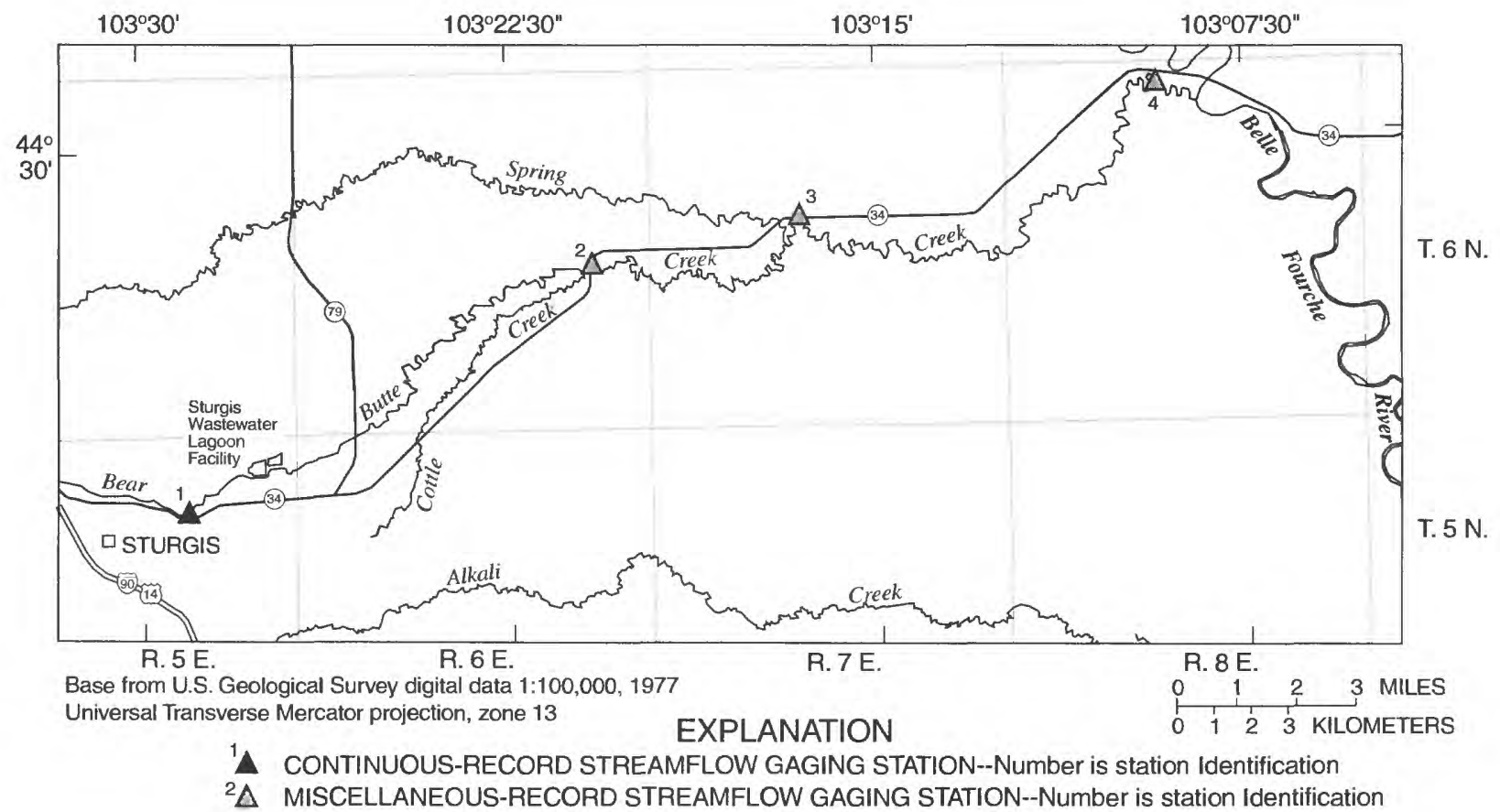

Figure 2. Locations of selected gaging stations and water-quality sampling sites. 
Table 2. Miscellaneous discharge measurements for selected streamflow-gaging stations $\left[--\right.$ no data; $\mathrm{ft}^{3} / \mathrm{s}$, cubic feet per second]

\begin{tabular}{|c|c|c|c|c|c|c|c|c|}
\hline Date & Time & $\begin{array}{c}\text { Bear Butte Creek } \\
\text { at Sturgis } \\
06437400 \\
(\text { site 1) } \\
\left(\mathrm{ft}^{3} / \mathrm{s}\right)\end{array}$ & Time & $\begin{array}{c}\text { Bear Butte Creek } \\
\text { below Sturgis } \\
442811103205000 \\
(\text { site } 2) \\
\left(\mathrm{ft}^{3} / \mathrm{s}\right)\end{array}$ & Time & $\begin{array}{c}\text { Bear Butte Creek } \\
\text { near Sturgis } \\
06437500 \\
(\text { site 3) } \\
\left(\mathrm{ft}^{3} / \mathrm{s}\right)\end{array}$ & Time & $\begin{array}{c}\text { Bear Butte Creek } \\
\text { near mouth, } \\
\text { near Vale } \\
443048103091400 \\
(\text { site 4) } \\
\left(\mathrm{ft}^{3} / \mathrm{s}\right)\end{array}$ \\
\hline 06-16-1998 & 1000 & 3.8 & 1145 & 4.2 & 1315 & 7.5 & 1455 & 7.3 \\
\hline 06-18-1998 & 1300 & 349 & 1405 & 52 & 1532 & 299 & 1745 & 529 \\
\hline 06-19-1998 & 1020 & 149 & -- & -- & -- & -- & -- & -- \\
\hline 06-20-1998 & 1230 & 83 & -- & -- & -- & -- & -- & -- \\
\hline $06-22-1998$ & 0950 & 42 & -- & -- & -- & -- & -- & - \\
\hline 06-25-1998 & 1057 & 19 & -- & - & -- & -- & - & $\cdots$ \\
\hline 06-30-1998 & 1100 & 5.8 & - & -- & -- & -- & -- & -- \\
\hline 07-07-1998 & 0915 & 4.7 & 1100 & 6.2 & 1240 & 12 & 1415 & 12 \\
\hline 08-11-1998 & 1015 & 4.4 & 1215 & 3.8 & 1340 & 3.5 & 1450 & 3.1 \\
\hline 09-16-1998 & 0855 & 3.5 & 1100 & 1.1 & 1230 & 1.1 & 1245 & 0 \\
\hline $10-06-1998$ & 1040 & 7.3 & 1345 & 17 & -- & -- & - & -- \\
\hline $10-07-1998$ & -- & -- & -- & -- & 1035 & 48 & 1330 & 56 \\
\hline $11-17-1998$ & 0930 & 14 & 1130 & 22 & 1250 & 51 & 1420 & 62 \\
\hline $12-15-1998$ & 0930 & 6.5 & 1115 & 10 & 1250 & 20 & 1430 & 22 \\
\hline 01-25-1999 & 1125 & 4.8 & 1300 & 8.4 & -- & -- & - & - \\
\hline 01-26-1999 & -- & -- & -- & -- & 0855 & 15 & 1125 & 14 \\
\hline 02-10-1999 & 0915 & 4.3 & 1055 & 6.6 & 1230 & 18 & 1405 & 21 \\
\hline 03-09-1999 & 0915 & 3.8 & 1105 & 6.3 & 1245 & 18 & 1435 & 20 \\
\hline 04-06-1999 & 0900 & 4.3 & 1055 & 20 & 1220 & 69 & 1400 & 107 \\
\hline 04-21-1999 & 1110 & 4.7 & 1200 & 15 & 1255 & 29 & 1350 & 42 \\
\hline 05-10-1999 & 0900 & 6.6 & 1130 & 16 & 1310 & 32 & 1445 & 37 \\
\hline 06-09-1999 & 0915 & 7.4 & 1100 & 17 & 1245 & 80 & 1430 & 119 \\
\hline 06-15-1999 & 0845 & 92 & 1027 & 122 & 1225 & 190 & 1456 & 265 \\
\hline 07-19-1999 & 0735 & 9.9 & 0925 & 14 & 1050 & 31 & 1210 & 32 \\
\hline 08-16-1999 & 0840 & 7.4 & 1025 & 6.8 & 1145 & 12 & 1255 & 12 \\
\hline 09-14-1999 & 0910 & 6.2 & 1100 & 5.2 & 1245 & 9.6 & 1430 & 7.9 \\
\hline $10-26-1999$ & 0910 & 4.2 & 1050 & 5.6 & 1220 & 10 & 1345 & 12 \\
\hline 11-16-1999 & 0850 & 3.8 & 1025 & 5.7 & 1155 & 10 & 1325 & 11 \\
\hline 12-07-1999 & 0900 & 3.9 & 1050 & 5.5 & 1205 & 12 & 1340 & 16 \\
\hline $01-18-2000$ & 0905 & 3.5 & 1105 & 4.6 & 1255 & 9.4 & 1425 & 11 \\
\hline $02-22-2000$ & 0845 & 2.9 & 1030 & 4.5 & 1200 & 9.4 & 1330 & 13 \\
\hline $03-28-2000$ & 0900 & 3.2 & 1130 & 7.5 & 1405 & 19 & - & - \\
\hline $03-29-2000$ & - & -- & -- & -- & - & -- & 1000 & 20 \\
\hline $04-04-2000$ & 0855 & 3.0 & 1035 & 6.3 & 1200 & 14 & 1330 & 13 \\
\hline $05-09-2000$ & 0900 & 4.6 & 1030 & 16 & 1215 & 36 & 1345 & 53 \\
\hline $05-18-2000$ & 0900 & 7.7 & 1100 & 19 & 1230 & 33 & 1400 & 37 \\
\hline
\end{tabular}


Bear Butte Creek at Sturgis, 06437400, Site 1
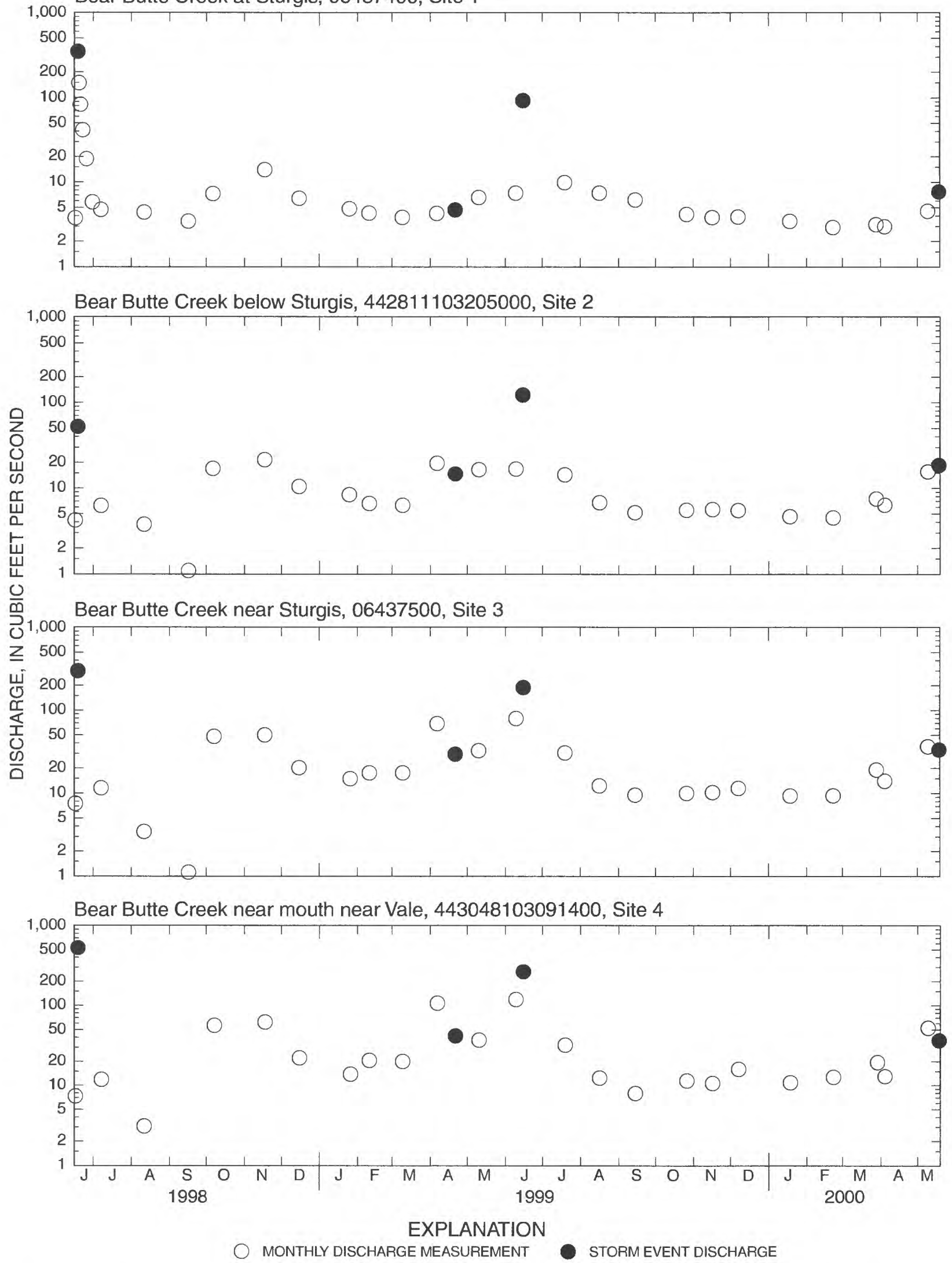

Figure 3. Miscellaneous discharge measurements for selected streamflow gaging stations, May 1998 to May 2000. 
Continuous stage was recorded at site 1 , Bear Butte Creek at Sturgis (fig. 4). Daily discharge was calculated based on measured discharge and continuous stage recordings and is published annually by water year (October to September) in the Water Resources Data Report for South Dakota (U.S. Geological Survey, 1999, 2000). Flow data collected from October 1999 through September 2000 will be published in the water year 2000 Annual Data Report. For storm events, an effort was made to determine discharge and collect water-quality samples during either the first flush (rise of the hydrograph) or near the peak of the event (fig. 4).

Figure 5 presents a comparison of the discharge measurements from this study at site 3, Bear Butte Creek near Sturgis, with the long-term statistical range of flows during 1945-72. This site has numerous periods of zero flow so the minimum line on the plot corresponds with the $\mathrm{x}$-axis as does the 25 th percentile for much of the late summer and fall. All measurements in 1998-2000 except one exceed the 1945-72 median and most approach or exceed the 75th percentile.

\section{DESCRIPTION OF WATER-QUALITY DATA}

This section presents the water-quality data from samples collected at the same time as the monthly and storm-event discharge measurements. A discussion of QA/QC sampling, as well as beneficial-use and waterquality criteria are presented.

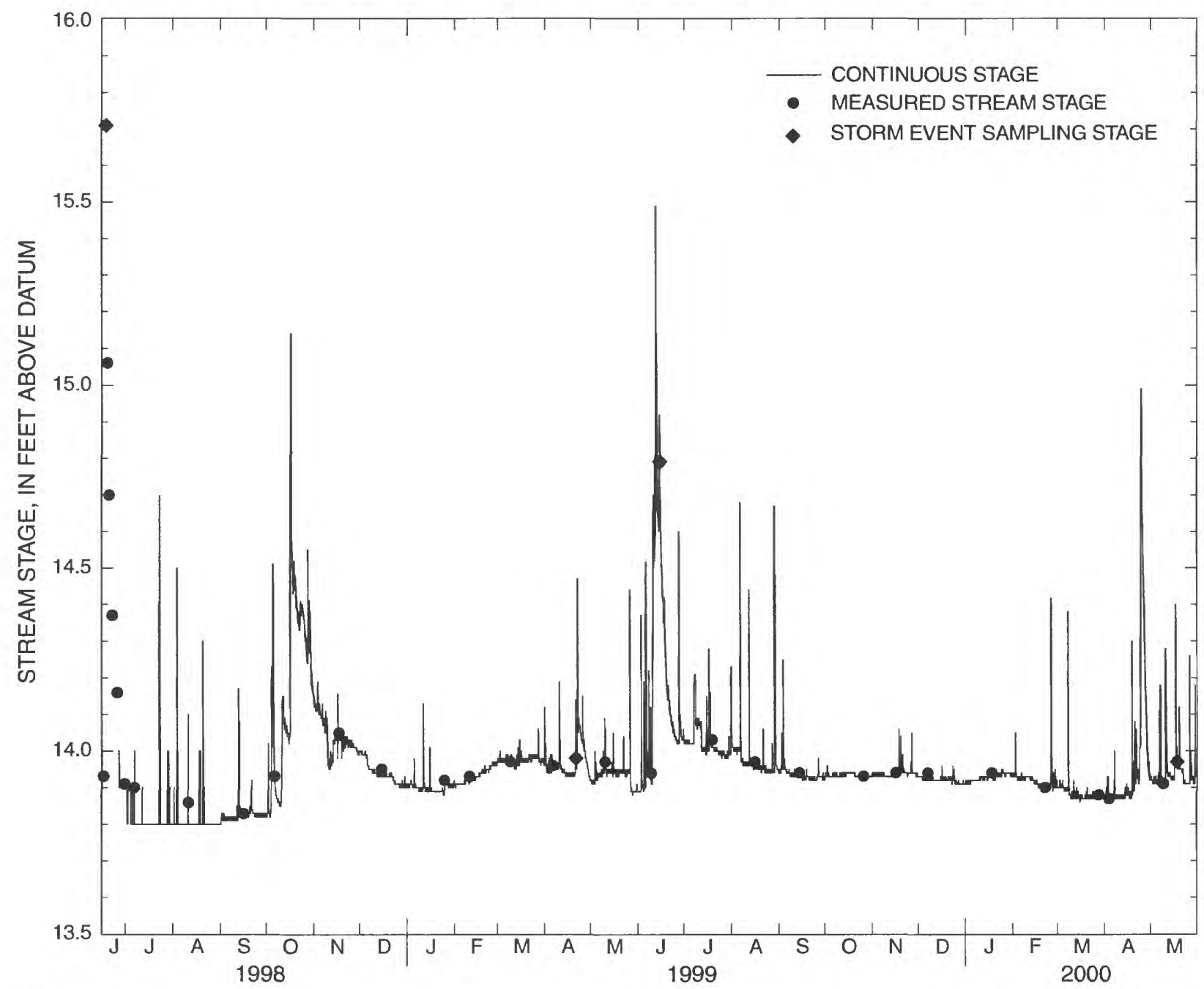

Figure 4. Continuous stage recordings, stage at miscellaneous measurements, and storm-event stage at Bear Butte Creek at Sturgis, 06437400. 


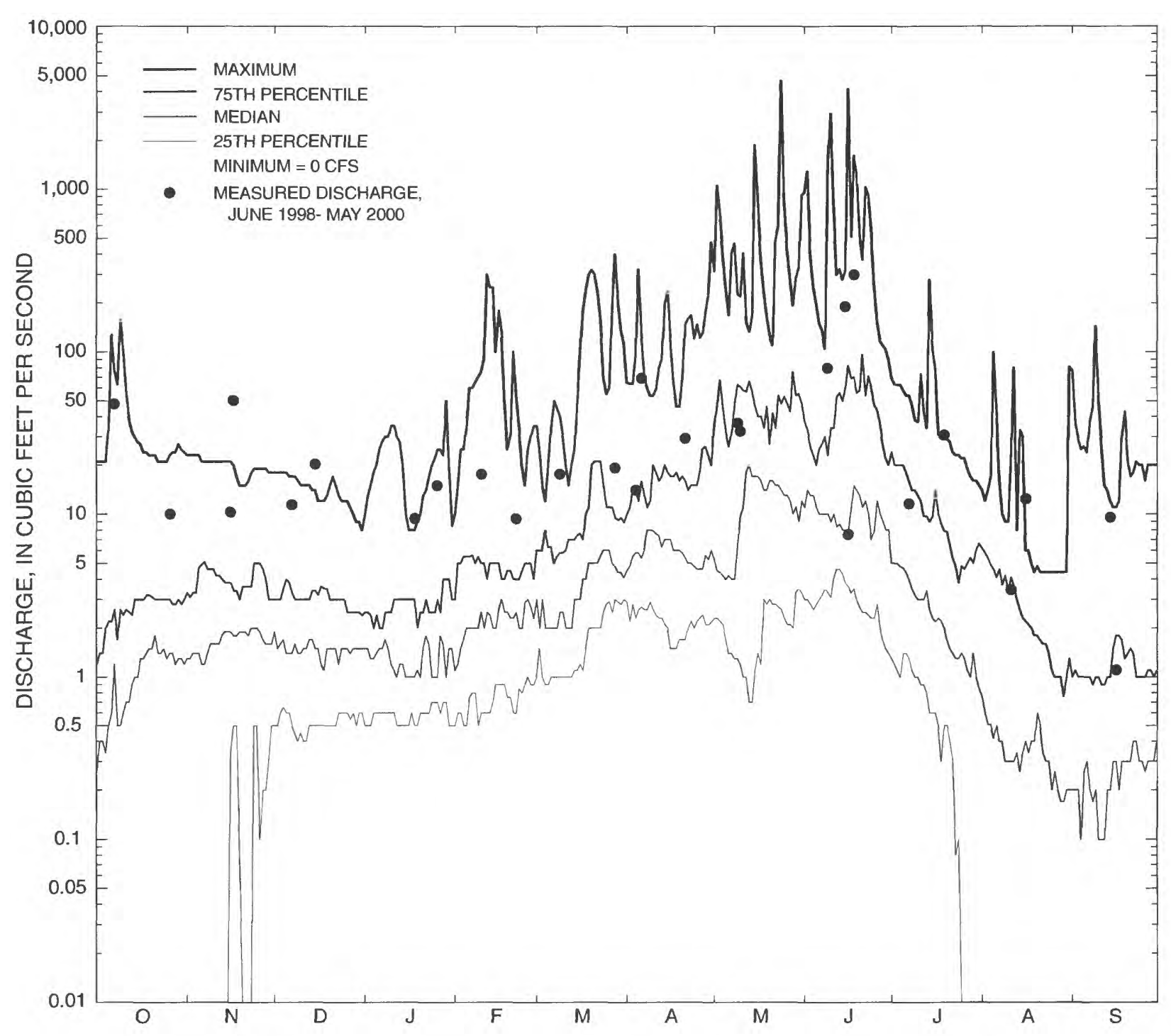

Figure 5. Comparison of miscellaneous discharge measurements during water years 1998-2000 to hydrographs of long-term statistical range of flows (1945-72) at Bear Butte Creek near Sturgis, 06437500

\section{Water-Quality Results}

Water-quality results are presented in table 6 in the Supplemental Information section at the end of the report and include data analyzed by the SHL as well as QA/QC data analyzed by both the SHL and NWQL. Discharge and field measurements followed standard USGS methods (Rantz and others, 1982). Sampling methods and criteria for water-quality sampling followed DENR's Water Quality 106 Quality Assurance Project Plan (1988). An additional 10 percent of samples were collected for QA/QC. Included in the
QA/QC were samples collected using standard USGS techniques (Horowitz and others, 1994) and sent to the NWQL for analysis.

Trace-element results from the first two analyses at each site should be used with caution due to contamination (see following section for details). Ion analyses were switched from total to dissolved concentrations in December 1998. Boxplots and trilinear diagrams are presented to provide indications of changes in water quality within the basin below the City of Sturgis (figs. 6 and 7). 

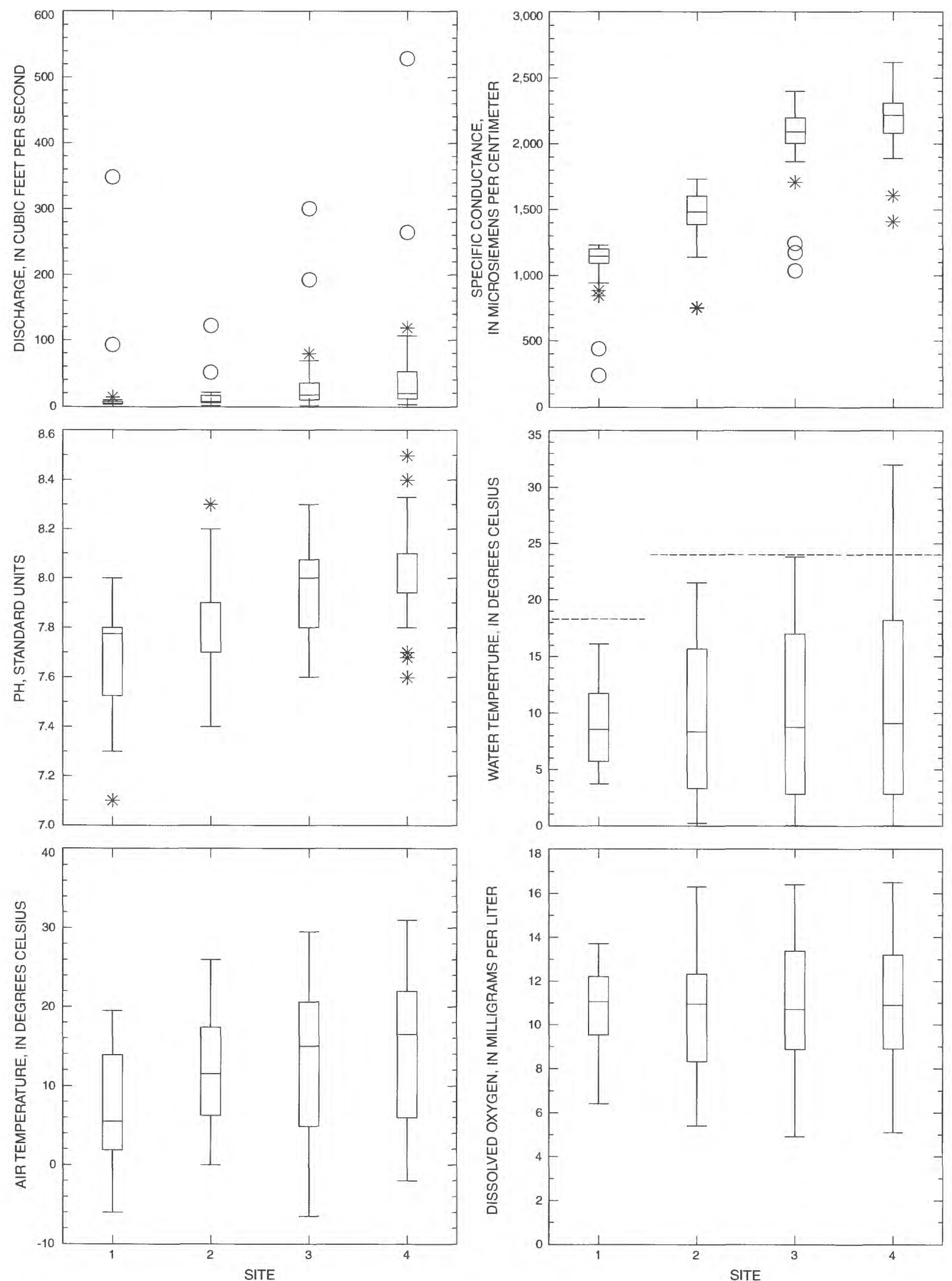

Figure 6. Boxplots by site for selected constituents for Bear Butte Creek from June 1998 to May 2000. 

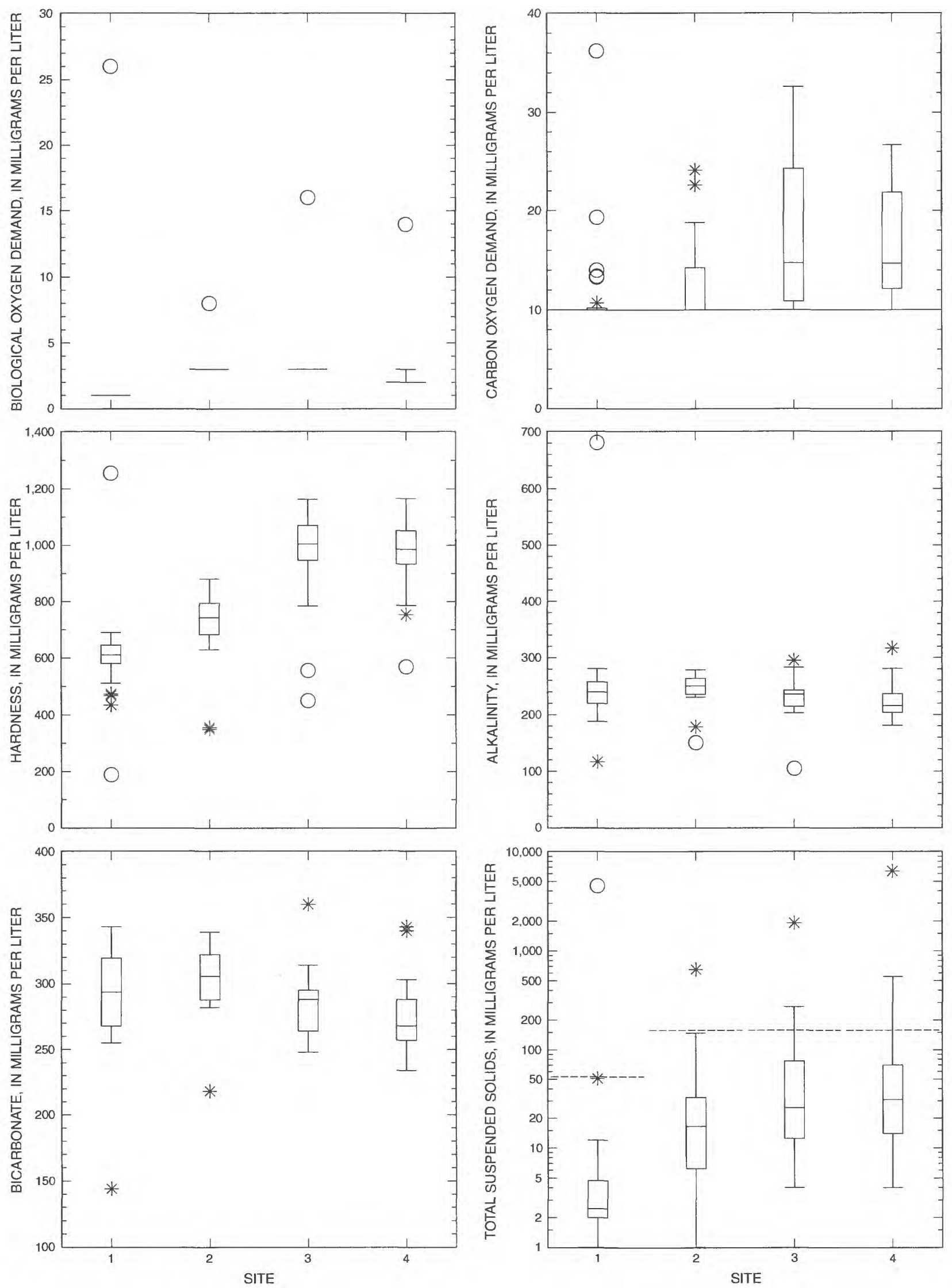

Figure 6. Boxplots by site for selected constituents for Bear Butte Creek from June 1998 to May 2000.--Continued 

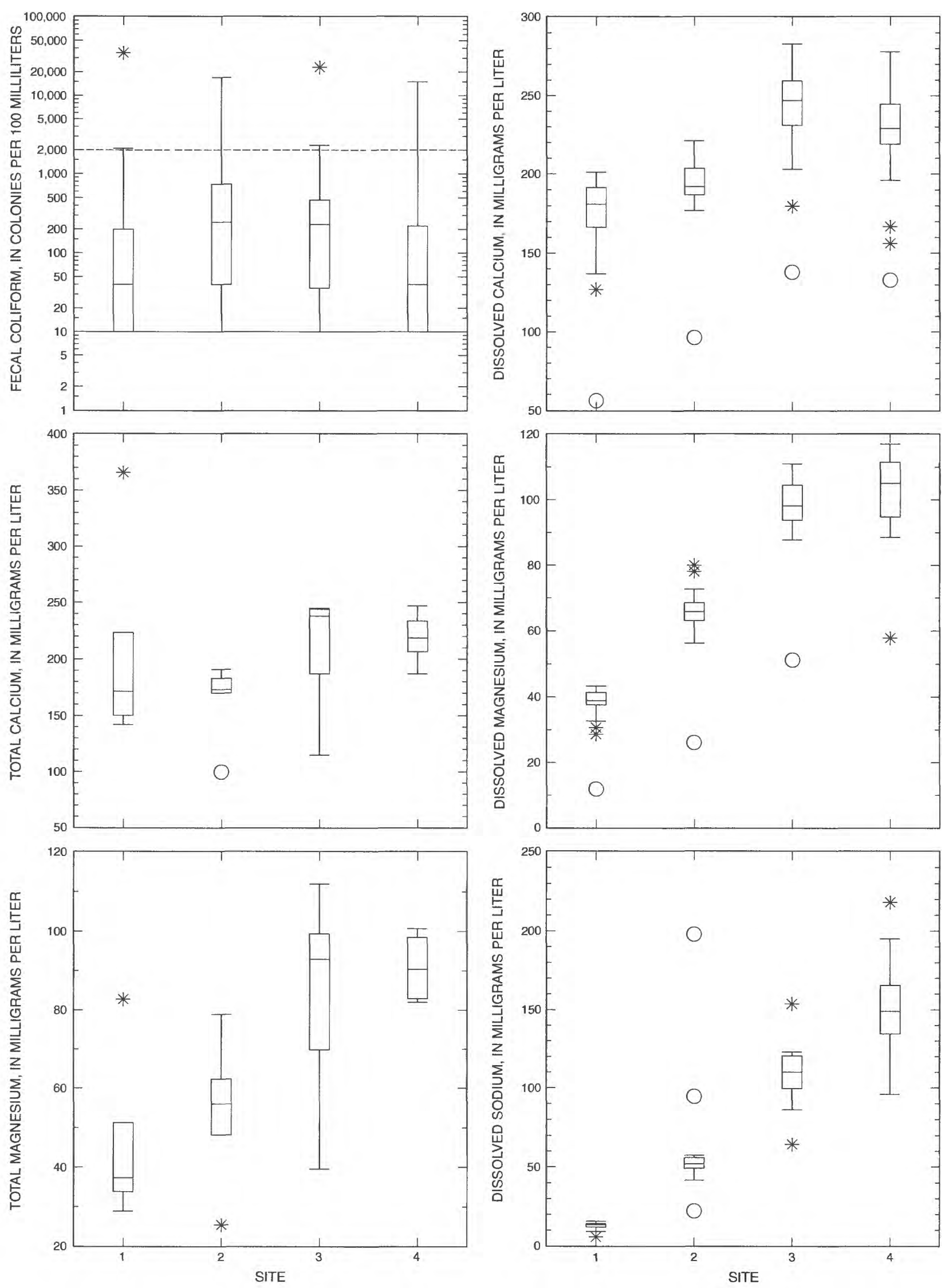

Figure 6. Boxplots by site for selected constituents for Bear Butte Creek from June 1998 to May 2000.--Continued 

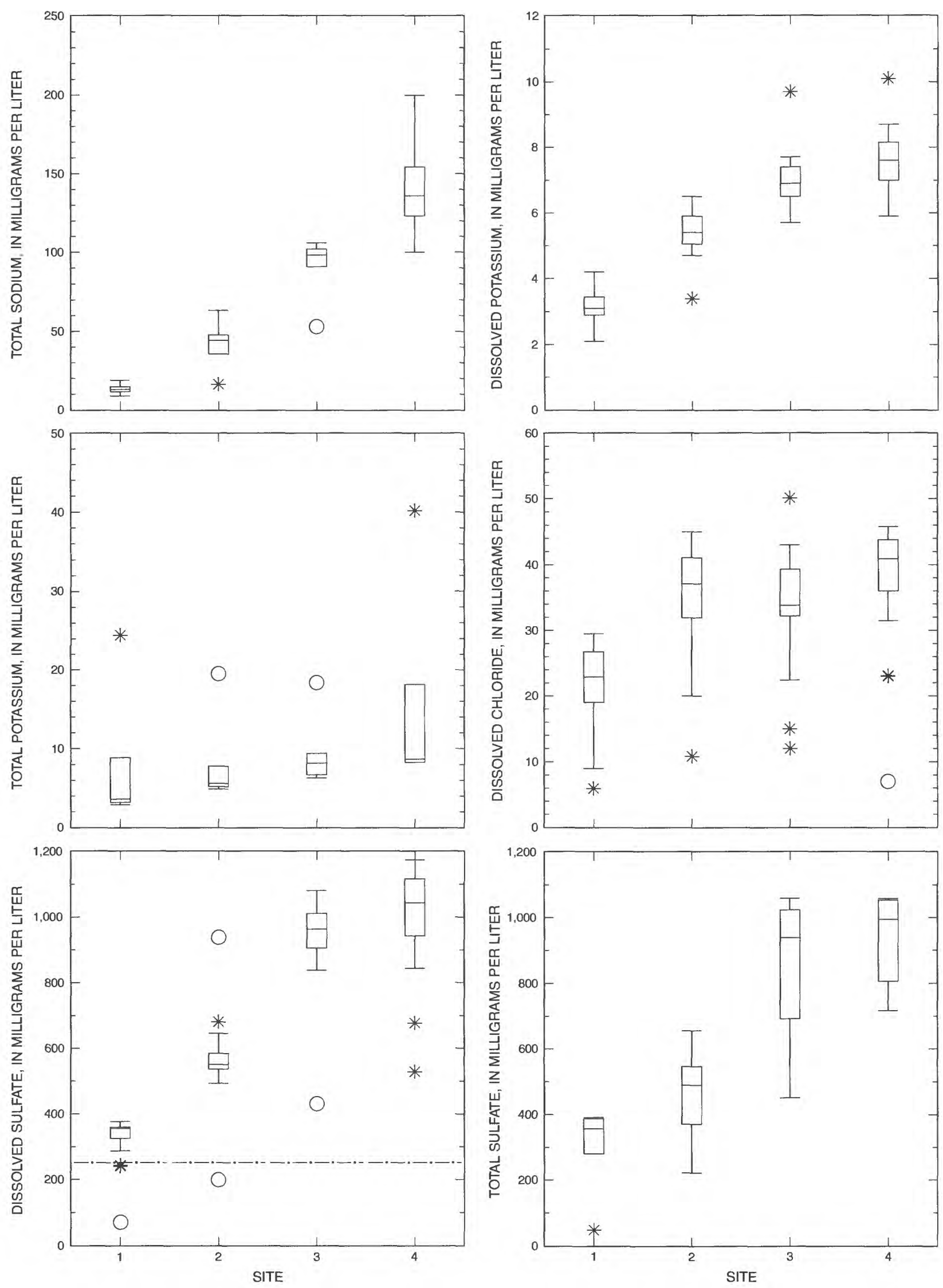

Figure 6. Boxplots by site for selected constituents for Bear Butte Creek from June 1998 to May 2000.--Continued 

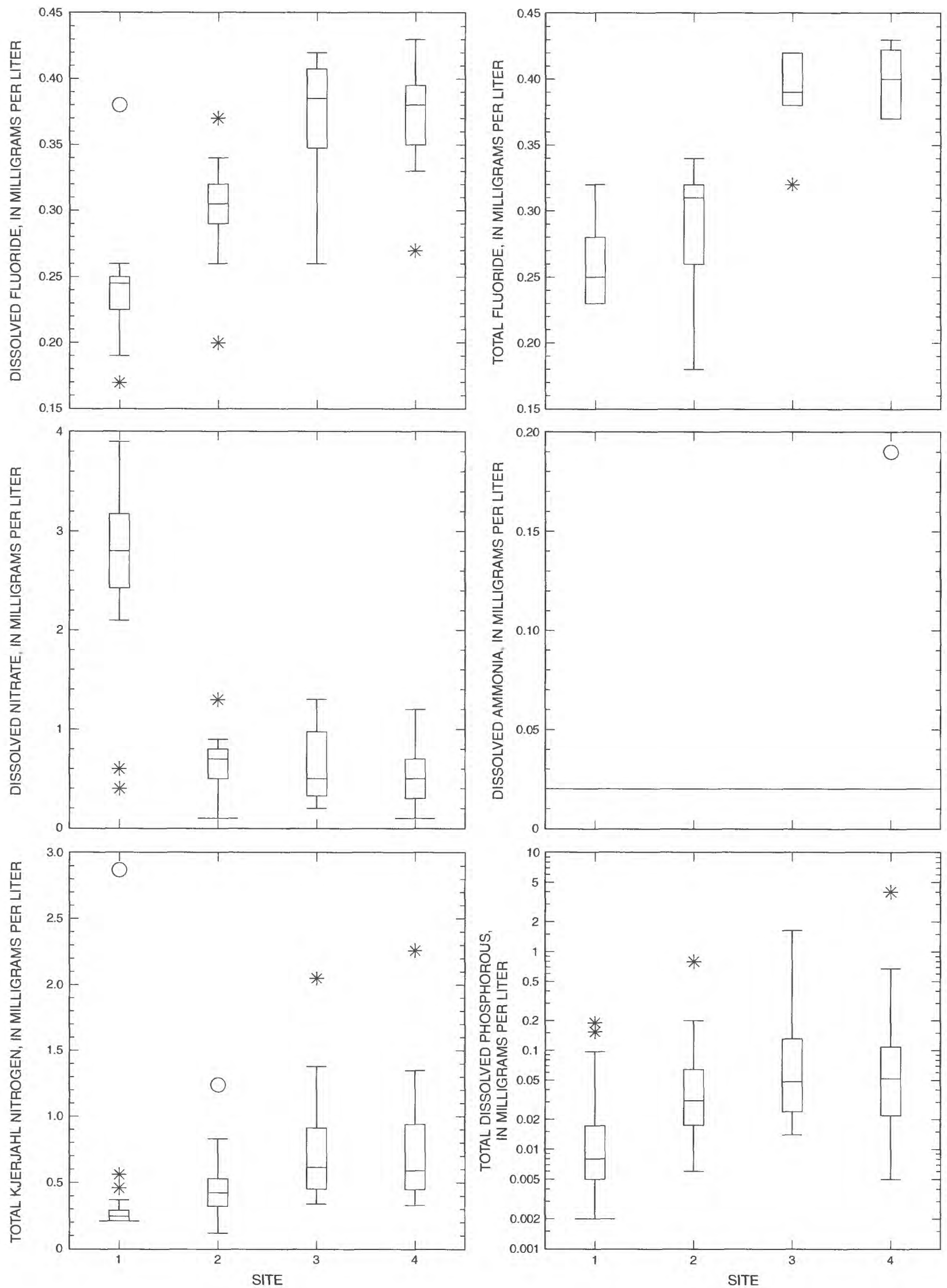

Figure 6. Boxplots by site for selected constituents for Bear Butte Creek from June 1998 to May 2000.--Continued 

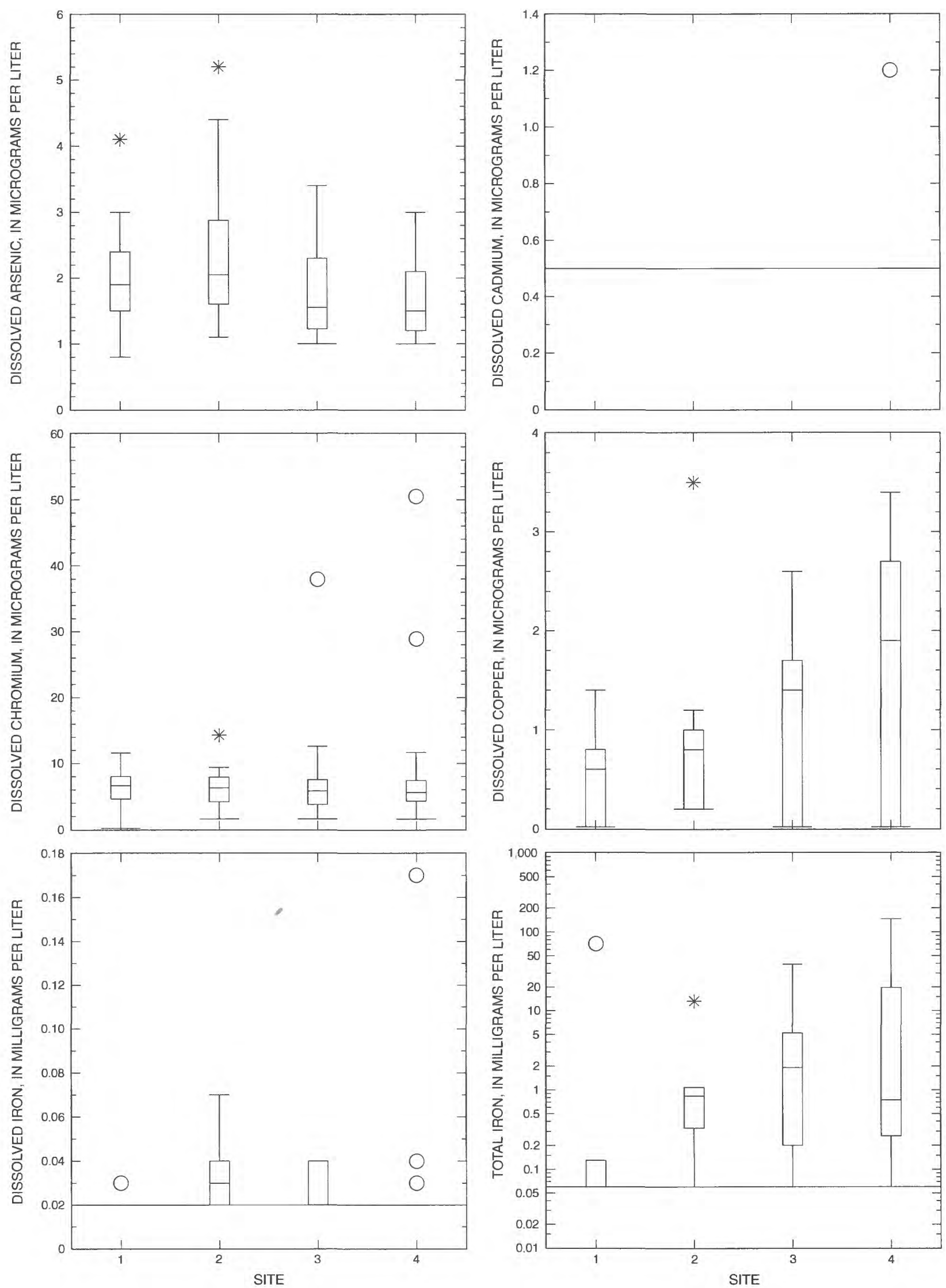

Figure 6. Boxplots by site for selected constituents for Bear Butte Creek from June 1998 to May 2000.--Continued 

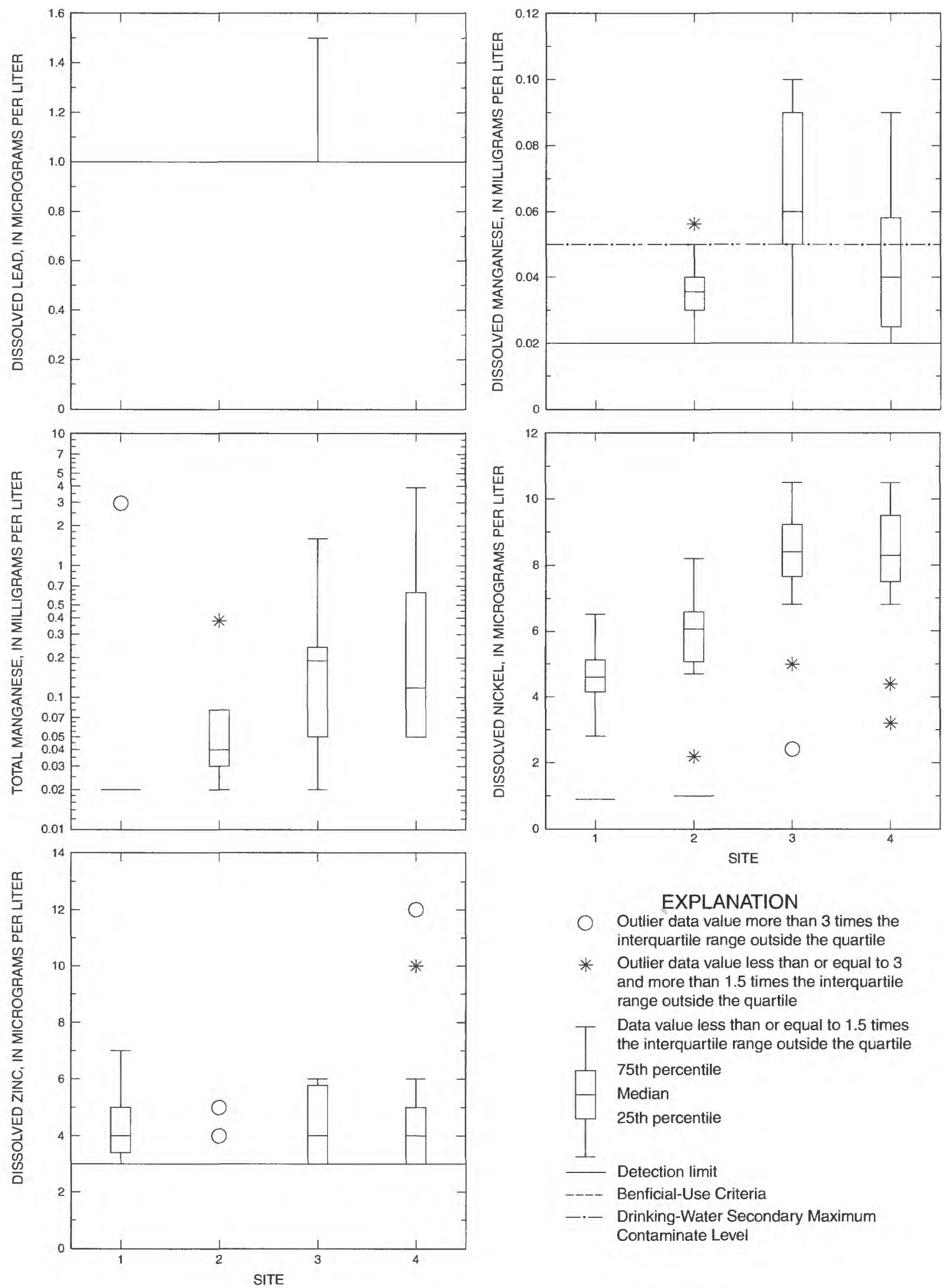

EXPLANATION

Outlier data value more than 3 times the interquartile range outside the quartile

* Outlier data value less than or equal to 3 and more than 1.5 times the interquartile range outside the quartile

- Data value less than or equal to 1.5 times the interquartile range outside the quartile 75th percentile

Median

25th percentile

Detection limit

Benficial-Use Criteria

Drinking-Water Secondary Maximum Contaminate Level

Figure 6. Boxplots by site for selected constituents for Bear Butte Creek from June 1998 to May 2000.--Continued 


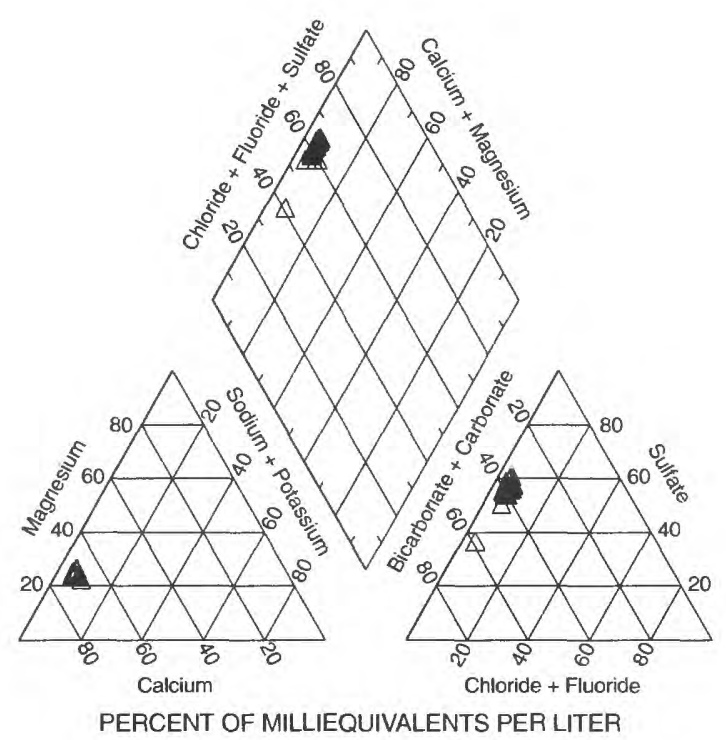

Bear Butte Creek at Sturgis, SD, 06437400, Site 1

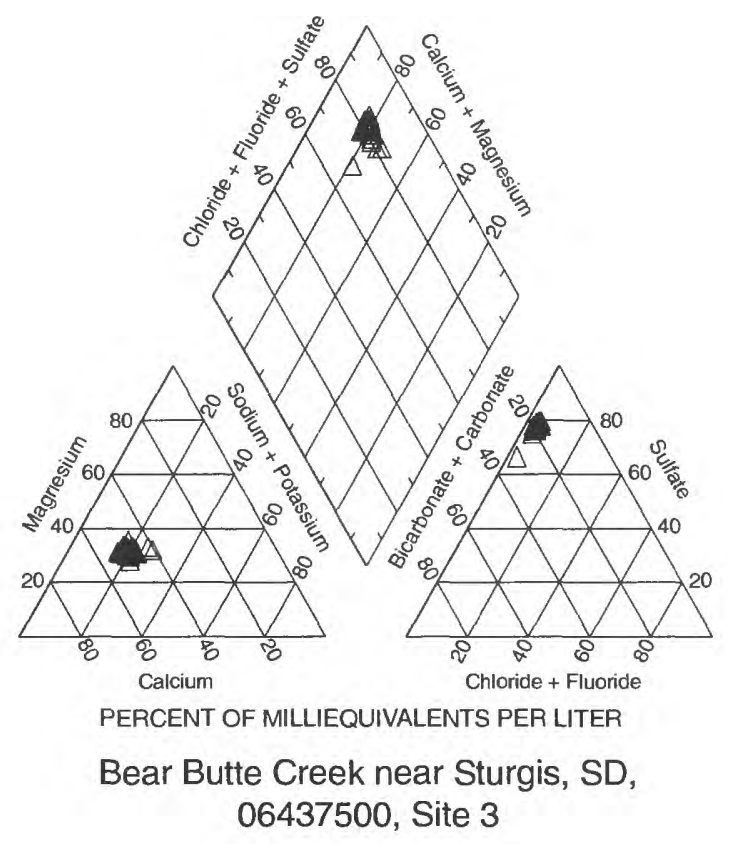

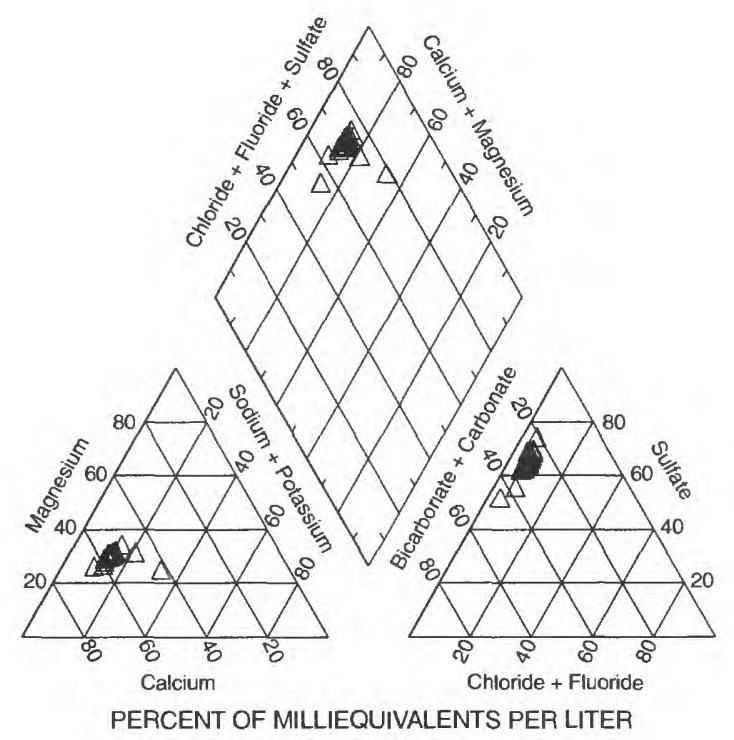

Bear Butte Creek below Sturgis, SD, 442811103205000 , Site 2

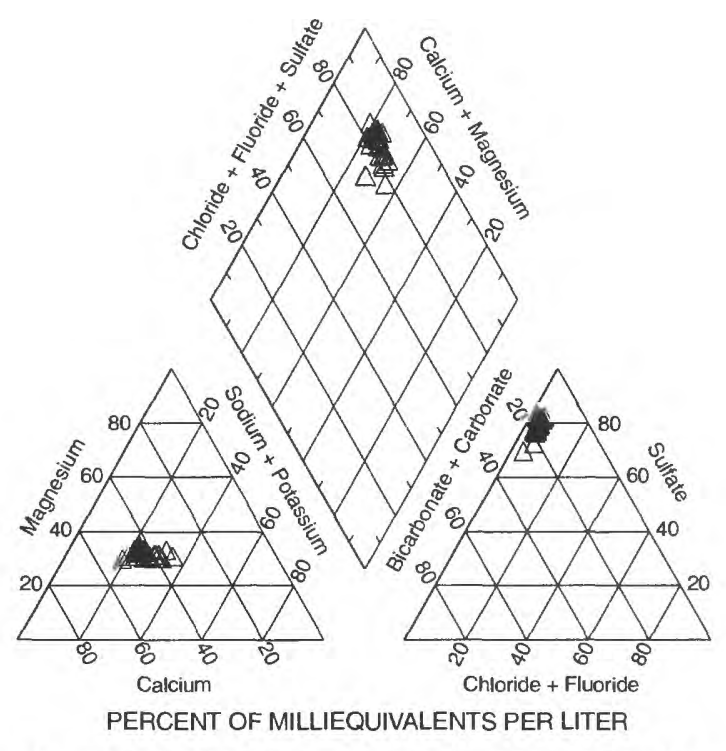

Bear Butte Creek near mouth, near Vale, SD, 443048103091400 , Site 4

Figure 7. Trilinear diagrams (Piper, 1944) showing proportional concentrations of major ions for select sites. 


\section{Quality-Assurance/Quality-Control Results}

An additional 10 percent of the water-quality samples were collected to examine QA/QC. QA/QC samples included splits and blanks and were sent for analysis at both the SHL and NWQL (table 3).

Following the analysis of the first two sample sets, the SHL had concerns about the cleaning method used for the dissolved trace-element analysis. Bottles were cleaned using chromic acid and contamination of chromium was suspected. New sample bottles were used for subsequent sampling. In an effort to obtain indications of possible contamination levels, two blank samples were analyzed (site 1 and site 2, Oct. 6, 1998) using the chromic-acid cleaned bottles. Results from the blanks indicated that contamination was probably taking place for arsenic, chromium, nickel, and zinc. Therefore, trace-element results from the first two analyses at each site should be used with caution.
Splits analyzed by the SHL were very similar with percent difference less than 5 percent for most constituents (table 6). Those with over 5-percent difference were generally at or near the laboratory reporting limit.

Splits analyzed at the NWQL showed the most variability from SHL data for fecal coliform and alkalinity at site 1 on October 6, 1998. Some constituents can vary a great deal with slight changes in location, and differences may be related to sampling methods. Nutrient concentrations generally were smaller in the NWQL results, again possibly due to sampling methods.

For the March 28, 2000, sampling, rather significant differences were noted for chromium and nickel in the NWQL and SHL splits (sites 2 and 3), with results from NWQL at or near the laboratory reporting limits and SHL results ranging from 5 to $8 \mu \mathrm{g} / \mathrm{L}$ (micrograms per liter) higher. In an effort to eliminate sampling method, bottles, and preservation methods as the cause of difference, a split was sent to

Table 3. Description of quality-assurance/quality-control samples

[SHL, South Dakota State Health Laboratory; NWQL, U.S. Geological Survey National Water Quality Laboratory]

\begin{tabular}{|c|c|c|c|}
\hline Date & Time & Site & Type of sample \\
\hline $10-06-1998$ & 1041 & 06437400 & $\begin{array}{l}\text { SHL, blank to test chromium contamination in bottles, } \\
\text { monthly site sample at } 1040\end{array}$ \\
\hline $10-06-1998$ & 1048 & 06437400 & NWQL, split of 1040 monthly sample \\
\hline $10-06-1998$ & 1345 & 442811103205000 & $\begin{array}{l}\text { SHL, blank to test chromium contamination in bottles. } \\
\text { monthly site sample at } 1346\end{array}$ \\
\hline $10-06-1998$ & 1350 & 442811103205000 & NWQL, split of 1346 monthly sample \\
\hline 10-07-1998 & 1036 & 06437500 & SHL, split of 1035 monthly sample \\
\hline $10-07-1998$ & 1331 & 443048103091400 & SHL, split of 1330 monthly sample \\
\hline $03-28-2000$ & 1135 & 442811103205000 & NWQL, split of 1130 monthly sample \\
\hline $03-28-2000$ & 1402 & 06437500 & SHL, split of 1400 monthly sample \\
\hline $03-28-2000$ & 1405 & 06437500 & NWQL, split of 1400 monthly sample \\
\hline $05-18-2000$ & 0905 & 06437400 & SHL, split of 0900 storm-event sample \\
\hline $05-18-2000$ & 0908 & 06437400 & $\begin{array}{l}\text { SHL, split of } 0900 \text { storm-event sample using USGS } \\
\text { analysis bottle and acid to address differenced in } \\
\text { chromium and nickel between NWQL and SHL }\end{array}$ \\
\hline
\end{tabular}


the SHL to look at within-laboratory variability and a third sample was analyzed just for trace elements. The third sample was collected using USGS methods, bottles, and preservation methods, but analyzed at the SHL. Slight variability between the 3 samples was noted for chromium, with concentrations ranging from 9.8 to $11.9 \mu \mathrm{g} / \mathrm{L}$, but differences were not of the same magnitude as the March 28 sample results. Laboratory digestion procedures and methods of analysis are the most likely cause of the concentration differences.

Values for alkalinity can vary due to holding times and storage conditions as well.

\section{Beneficial-Use Designations and Water-Quality Criteria}

General information is provided in this section to enable the reader to compare water-quality criteria to constituent concentrations measured in the lower reach of Bear Butte Creek from June 1998 to May 2000. Tables 4 and 5 present the beneficial use designations and water-quality standards for select sections of Bear Butte Creek. Figure 8 presents the relation between hardness and freshwater aquatic-life standards of selected trace elements.

Table 4. Beneficial-use designations for segments of Bear Butte Creek

\begin{tabular}{ll}
\hline \multicolumn{1}{c}{ Stream segment } & \multicolumn{1}{c}{ Beneficial-use designation } \\
\hline All of Bear Butte Creek & $\begin{array}{c}\text { Limited contact recreation waters; irrigation waters; wildlife } \\
\text { propagation and stock-watering waters }\end{array}$ \\
From Belle Fourche River to Highway 79 & Coldwater marginal fish life propagation waters \\
Highway 79 to Deadman Creek & Coldwater permanent fish life propagation waters \\
$\begin{array}{l}\text { Deadman Creek to Section 2, Township 4N, Range 4E } \\
\begin{array}{l}\text { Section 2, Township 4N, Range 4E to Section 22, Township } \\
\text { 4N, Range 3E }\end{array}\end{array}$ & Coldwater marginal fish life propagation waters \\
\hline
\end{tabular}


Table 5. Water-quality standards for selected physical properties and constituents

[All concentrations in milligrams per liter unless otherwise noted; MCL, maximum contaminate level; SMCL, secondary maximum contaminate level; $\mu$ S/ $\mathrm{cm}$, microsiemens per centimeter at $25^{\circ} \mathrm{C} ; \mathrm{mg} / \mathrm{L}$, milligrams per liter; $\mu \mathrm{g} / \mathrm{L}$, micrograms per liter; $\mathrm{mL}$, milliliter;

${ }^{\circ} \mathrm{C}$, degrees Celsius; --, not applicable or no data available]

\begin{tabular}{|c|c|c|c|c|c|c|c|c|}
\hline \multirow[b]{2}{*}{ Property or constituent } & \multicolumn{2}{|c|}{$\begin{array}{l}\text { U.S. Environmental } \\
\text { Protection Agency } \\
\text { drinking-water } \\
\text { standards }\end{array}$} & \multicolumn{5}{|c|}{ Beneficial-use criteria } & \multirow{2}{*}{$\begin{array}{l}\text { Aquatic-life } \\
\text { value for } \\
\text { fisheries } \\
\text { (acutel } \\
\text { chronic) } \\
\mu g / L)^{2}\end{array}$} \\
\hline & $\begin{array}{l}\text { Drinking } \\
\text { water } \\
\text { MCL }^{1}\end{array}$ & $\begin{array}{l}\text { Drinking } \\
\text { water } \\
\text { SMCL }^{1}\end{array}$ & $\begin{array}{l}\text { Coldwater } \\
\text { permanent } \\
\text { fish life } \\
\text { propagation } \\
\text { waters }^{2}\end{array}$ & $\begin{array}{c}\text { Coldwater } \\
\text { marginal } \\
\text { fish life } \\
\text { propagation } \\
\text { waters }^{2}\end{array}$ & $\begin{array}{l}\text { Limited } \\
\text { contact } \\
\text { recreation } \\
\text { waters }^{2}\end{array}$ & $\begin{array}{l}\text { Wildlife } \\
\text { propagation } \\
\text { and stock- } \\
\text { watering } \\
\text { waters }^{2}\end{array}$ & $\begin{array}{l}\text { Irrigation } \\
\text { waters }^{2}\end{array}$ & \\
\hline $\begin{array}{l}\text { Specific conductance } \\
(\mu \mathrm{S} / \mathrm{cm})\end{array}$ & -- & - & - & -- & -- & $4,000 /^{3} 7,000$ & $2,500 / /^{3} 4,375$ & - \\
\hline $\mathrm{pH}$ range (standard units) & -- & $6.5-8.5$ & $6.6 / 8.6$ & $6.5-8.8$ & -- & $6.0-9.5$ & -- & -- \\
\hline Temperature $\left({ }^{\circ} \mathrm{C}\right)$ & -- & -- & 18.3 & 24 & -- & -- & -- & -- \\
\hline $\begin{array}{l}\text { Dissolved oxygen } \\
\text { (minimum concentration) }\end{array}$ & -- & - & 6.0 & 5.0 & 5.0 & -- & - & -- \\
\hline Total alkalinity (as $\mathrm{CaCO}_{3}$ ) & -- & -- & & & & $750 / /^{3} 1,313$ & & \\
\hline Total suspended solids & -- & -- & $30 / /^{3} 53$ & $90 /^{\beta} 158$ & -- & -- & - & -- \\
\hline Chloride & -- & 250 & $100 /^{3} 175$ & -- & -- & -- & - & -- \\
\hline Fluoride & 4 & 2 & -- & -- & -- & -- & -- & -- \\
\hline Sulfate & -- & 250 & -- & -- & -- & -- & -- & - \\
\hline Nitrate & 10 & -- & - & -- & -- & $50 / 388$ & -- & -- \\
\hline Cyanide & 0.2 & -- & 220 & 220 & -- & -- & -- & $22 / 5.2$ \\
\hline Arsenic & 0.05 & - & - & -- & -- & -- & -- & $360 / 190$ \\
\hline Cadmium & 0.005 & -- & - & - & -- & -- & -- & ${ }^{4} 3.7 /^{4} 1.0$ \\
\hline Copper & -- & 1.0 & -- & - & -- & -- & -- & ${ }^{4} 17 /^{4} 11$ \\
\hline Iron & -- & 0.3 & -- & -- & -- & -- & -- & -- \\
\hline Lead & -- & -- & -- & -- & -- & -- & -- & ${ }^{4} 65 /^{4} 2.5$ \\
\hline Manganese & - & 0.05 & - & - & - & -- & -- & -- \\
\hline Mercury & 0.002 & -- & -- & -- & -- & -- & -- & $2.1 / /^{5} 0.012$ \\
\hline Nickel & -- & -- & - & - & -- & -- & -- & ${ }^{4} 1,400 /{ }^{4} 610$ \\
\hline Silver & -- & 0.1 & -- & -- & -- & -- & -- & ${ }^{4} 3.4 /-$ \\
\hline Zinc & -- & 5.0 & -- & -- & -- & -- & - & ${ }^{4} 110 /^{4} 100$ \\
\hline $\begin{array}{l}\text { Fecal coliform, per } 100 \mathrm{~mL} \\
\text { (mean/single sample) }\end{array}$ & -- & -- & - & -- & $1,000 / 32,000$ & -- & -- & -- \\
\hline
\end{tabular}

\footnotetext{
${ }^{1}$ U.S. Environmental Protection Agency, 2000

${ }^{2}$ South Dakota Department of Environment and Natural Resources, 1998

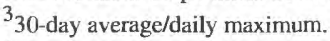

${ }^{4} \mathrm{Hardness}$-dependent criteria, value given is an example based on $\mathrm{CaCO}_{3}$ concentration of $100 \mathrm{mg} / \mathrm{L}$

${ }^{5}$ Chronic criteria based on total recoverable concentration.
} 


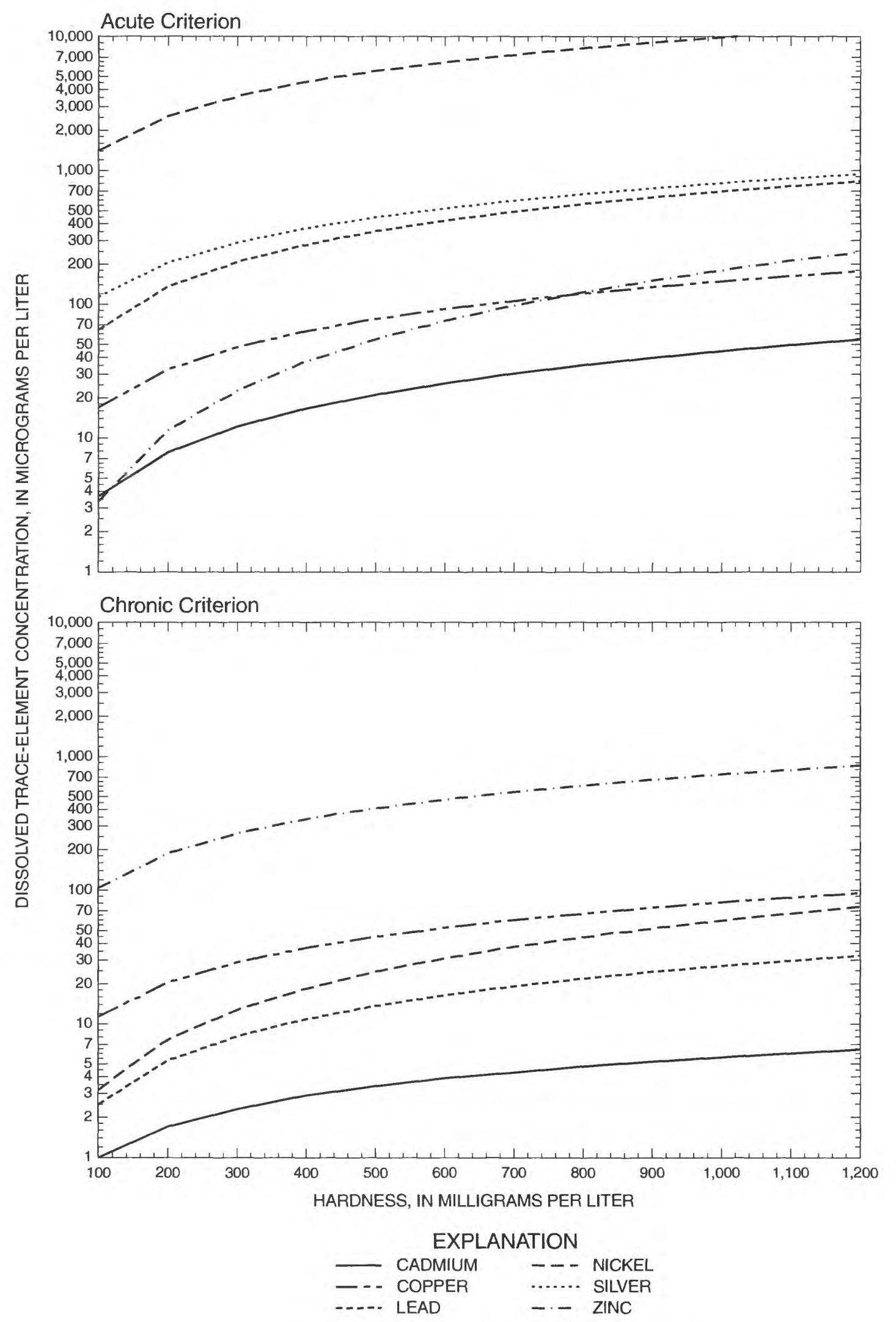

Figure 8. Relation between water hardness and freshwater aquatic-life standards for acute and chronic toxicity of selected trace elements (South Dakota Department of Environment and Natural Resourses, 1998). 


\section{REFERENCES}

Horowitz, A.J., Demas, C.R., Fitzgerald, K.K., Miller, T.L., and Rickert, D.A., 1994, U.S. Geological Survey protocol for the collection and processing of surface-water samples for the subsequent determination of inorganic constituents in filtered water: U.S. Geological Survey Open-File Report 94-539, $57 \mathrm{p}$.

Piper, A.M., 1944, A graphical procedure in the geochemical interpretation of water analyses: American Geophysical Union Transactions, v. 25, p. 914-923.

Rantz, S.E., and others, 1982, Measurement and computation of streamflow: U.S. Geological Survey WaterSupply Paper 2175, 2 v., 631 p.

South Dakota Department of Environment and Natural Resources, 1988, 106 Quality Assurance Project Plan. unnumbered document.
1998, Surface water quality-Administrative rules of South Dakota, 74:51, accessed August 14, 1998, at URL http://legis.state.sd.us/rules/rules/7451.htm.

Strobel, M.L., Sawyer, J.R., Jarrell, G.J., Schleicher, J.R., and Fahrenbach, M.D., 1999, Distribution of hydrologic units in the Black Hills area, South Dakota: U.S. Geological Survey Hydrologic Investigations Atlas HA-743, 3 sheets, scale 1:100,000.

U.S. Geological Survey, 1999-2000, Water resources data, South Dakota, water years 1998-99: U.S. Geological Survey Water Data Reports SD-98-1 to SD-99-1 (published annually).

U.S. Environmental Protection Agency, 2000, Current drinking water standards: Washington, D.C., Office of Groundwater and Drinking Water, accessed August 31, 2000, at URL http://www.epa.gov/OGWDW/mcl.html. 
SUPPLEMENTAL DATA 
Table 6. Water-quality data for selected sites along Bear Butte Creek

$\left[\mathrm{ft}^{3} / \mathrm{s}\right.$, cubic feet per second; $\mu \mathrm{S} / \mathrm{cm}$, microsiemens per centimeter; deg $\mathrm{C}$, degrees Celsius; $\mathrm{mg} / \mathrm{L}$, milligrams per liter; $\mu \mathrm{g} / \mathrm{L}$, micrograms per liter; cols, colonies; $\mathrm{mL}$, milliliters; $\mathrm{K}$, non-ideal colony count; <, less than; --, no data available]

\begin{tabular}{|c|c|c|c|c|c|c|c|c|c|c|c|}
\hline Date & Time & $\begin{array}{c}\text { Dis- } \\
\text { charge } \\
\left(\mathrm{ft}^{3} / \mathrm{s}\right)\end{array}$ & $\begin{array}{c}\text { Specific } \\
\text { conduc- } \\
\text { tance } \\
(\mu \mathrm{S} / \mathrm{cm})\end{array}$ & $\begin{array}{c}\mathrm{pH} \\
\text { (standard } \\
\text { units) }\end{array}$ & $\begin{array}{c}\text { Water } \\
\text { tempera- } \\
\text { ture } \\
(\operatorname{deg} C)\end{array}$ & $\begin{array}{c}\text { Air } \\
\text { tempera- } \\
\text { ture } \\
\text { (deg C) }\end{array}$ & $\begin{array}{c}\text { Dis- } \\
\text { solved } \\
\text { oxygen } \\
\text { (mg/L) }\end{array}$ & $\begin{array}{c}\text { Bio- } \\
\text { logical } \\
\text { oxygen } \\
\text { demand } \\
\text { (mg/L) }\end{array}$ & $\begin{array}{c}\text { Carbon } \\
\text { oxygen } \\
\text { demand } \\
(\mathrm{mg} / \mathrm{L})\end{array}$ & $\begin{array}{c}\text { Hardness } \\
\text { as } \mathrm{CaCO}_{3} \\
(\mathrm{mg} / \mathrm{L})\end{array}$ & $\begin{array}{l}\text { Labora- } \\
\text { tory } \\
\text { alkalinity } \\
\text { (mg/L) }\end{array}$ \\
\hline \multicolumn{12}{|c|}{ Site 1 - 06437400 Bear Butte Creek at Sturgis, SD } \\
\hline 06-16-1998 & 0930 & 3.8 & 1.130 & 7.8 & 11.5 & 13.5 & 11.9 & $<1$ & 7.4 & 640 & 241 \\
\hline $06-18-1998$ & 1300 & 349 & 239 & 7.8 & 11.0 & 11.0 & 9.6 & 26 & 36.2 & 1,255 & 681 \\
\hline 07-07-1998 & 0915 & 4.7 & 1,120 & 7.8 & 13.5 & 19.0 & 11.4 & $<1$ & 19.3 & 588 & 239 \\
\hline $08-11-1998$ & 1015 & 4.4 & 1,150 & 7.9 & 16.0 & 18.5 & 11.1 & $<1$ & 13.4 & 599 & 239 \\
\hline 09-16-1998 & 0855 & 3.5 & 1,200 & 7.8 & 13.5 & 14.0 & 9.5 & $<1$ & 4.41 & 610 & 245 \\
\hline $10-06-1998$ & 1040 & 7.3 & 1,090 & 7.6 & 10.0 & 8.0 & 10.1 & $<1$ & 1.79 & 528 & 196 \\
\hline${ }^{1} 10-06-1998$ & 1041 & 7.3 & 1,090 & 7.6 & 10.0 & 8.0 & 10.1 & -- & -- & -- & - \\
\hline${ }^{2} 10-06-1998$ & 1048 & 7.3 & 1,090 & 7.6 & 10.0 & 8.0 & 10.1 & -- & -- & -- & 253 \\
\hline $11-17-1998$ & 0930 & 14 & 941 & 7.4 & 6.5 & 3.0 & 11.9 & $<1$ & $<20$ & 473 & 209 \\
\hline $12-15-1998$ & 0930 & 6.5 & 1,120 & 7.3 & 6.0 & -3.0 & 12.3 & $<1$ & $<20$ & 601 & 260 \\
\hline 01-25-1999 & 1125 & 4.8 & 1,160 & 7.8 & 4.5 & .0 & 12.5 & $<1$ & $<10$ & 605 & 245 \\
\hline 02-10-1999 & 0915 & 4.3 & 1,160 & 7.8 & 5.5 & 3.0 & 13.7 & $<1$ & $<10$ & -- & 262 \\
\hline 03-09-1999 & 0915 & 3.8 & 1,150 & 7.7 & 3.5 & -1.5 & 13.3 & $<1$ & $<10$ & 580 & 221 \\
\hline 04-06-1999 & 0900 & 4.3 & 1,130 & 7.8 & 5.5 & 1.5 & 12.3 & $<1$ & $<10$ & 596 & 236 \\
\hline 04-21-1999 & 1040 & 4.7 & 1,010 & 7.9 & 9.0 & 8.0 & 11.0 & $<1$ & $<10$ & 511 & 219 \\
\hline 05-10-1999 & 0900 & 6.6 & 843 & 7.3 & 8.0 & 7.0 & 7.9 & $<1$ & 10.7 & 435 & 188 \\
\hline 06-09-1999 & 0915 & 7.4 & 1,090 & 8.0 & 12.0 & 14.5 & 9.3 & $<1$ & $<10$ & 580 & 235 \\
\hline 06-15-1999 & 0755 & 92 & 438 & 7.8 & 12.0 & 15.0 & 6.4 & $<1$ & $<10$ & 189 & 118 \\
\hline 07-19-1999 & 0735 & 9.9 & 1,200 & 7.3 & 14.0 & 19.5 & 8.5 & $<1$ & 13.3 & 640 & 267 \\
\hline 08-16-1999 & 0840 & 7.4 & 1,210 & 7.5 & 14.0 & 17.5 & 9.0 & $<1$ & $<10$ & 690 & 272 \\
\hline 09-14-1999 & 0910 & 6.2 & 1,230 & 7.4 & 11.5 & 5.0 & 8.9 & $<1$ & $<10$ & 670 & 250 \\
\hline $10-26-1999$ & 0910 & 4.2 & 1,220 & 7.6 & 9.5 & 6.0 & 9.7 & $<1$ & $<10$ & 658 & 248 \\
\hline $11-16-1999$ & 0850 & 3.8 & 1,200 & 7.7 & 8.5 & 3.0 & 11.2 & $<1$ & $<10$ & 653 & 281 \\
\hline $12-07-1999$ & 0900 & 3.9 & 1,210 & 7.1 & 6.5 & -.5 & 11.8 & $<1$ & $<10$ & 641 & 248 \\
\hline $01-18-2000$ & 0905 & 3.5 & 1,200 & 7.9 & 4.0 & -6.0 & 10.0 & $<1$ & $<10$ & 644 & 268 \\
\hline $02-22-2000$ & 0845 & 2.9 & 1,200 & 7.8 & 5.5 & 4.5 & 11.0 & $<1$ & $<10$ & 624 & 230 \\
\hline $03-28-2000$ & 0900 & 3.2 & 1,190 & 7.7 & 6.0 & 4.0 & 12.2 & $<1$ & $<10$ & 645 & 232 \\
\hline 04-04-2000 & 0855 & 3.0 & 1,190 & 7.6 & 5.0 & .0 & 10.7 & $<1$ & $<10$ & 649 & 251 \\
\hline $05-09-2000$ & 0900 & 4.6 & 1,120 & 8.0 & 8.5 & 10.0 & 12.2 & $<1$ & $<10$ & 611 & 220 \\
\hline $05-18-2000$ & 0900 & 7.7 & 885 & 8.0 & 8.0 & 4.0 & 12.8 & $<1$ & 14 & 468 & 215 \\
\hline${ }^{3} 05-18-2000$ & 0905 & 7.7 & 885 & 8.0 & 8.0 & 4.0 & 12.8 & $<1$ & $<10$ & 475 & 213 \\
\hline${ }^{3} 05-18-2000$ & 0908 & 7.7 & 885 & 8.0 & 8.0 & 4.0 & 12.8 & - & -- & -- & -- \\
\hline
\end{tabular}




\begin{tabular}{|c|c|c|c|c|c|c|c|c|c|c|c|}
\hline $\begin{array}{c}\text { Bicar- } \\
\text { bonate as } \\
\mathrm{CaCO}_{3} \\
(\mathrm{mg} / \mathrm{L})\end{array}$ & $\begin{array}{l}\text { Total } \\
\text { sus- } \\
\text { pended } \\
\text { solids } \\
\text { (mg/L) }\end{array}$ & $\begin{array}{l}\text { Fecal } \\
\text { coliform } \\
\text { (cols/ } \\
100 \mathrm{~mL} \text { ) }\end{array}$ & $\begin{array}{l}\text { Dis- } \\
\text { solved } \\
\text { calcium } \\
\text { (mg/L) }\end{array}$ & $\begin{array}{c}\text { Total } \\
\text { calcium } \\
\text { (mg/L) }\end{array}$ & $\begin{array}{l}\text { Dis- } \\
\text { solved } \\
\text { magne- } \\
\text { sium } \\
\text { (mg/L) }\end{array}$ & $\begin{array}{l}\text { Total } \\
\text { magne- } \\
\text { sium } \\
(\mathrm{mg} / \mathrm{L})\end{array}$ & $\begin{array}{l}\text { Dis- } \\
\text { solved } \\
\text { sodium } \\
\text { (mg/L) }\end{array}$ & $\begin{array}{l}\text { Total } \\
\text { sodium } \\
\text { (mg/L) }\end{array}$ & $\begin{array}{l}\text { Dis- } \\
\text { solved } \\
\text { potas- } \\
\text { sium } \\
\text { (mg/L) }\end{array}$ & $\begin{array}{l}\text { Total } \\
\text { potas- } \\
\text { sium } \\
\text { (mg/L) }\end{array}$ & $\begin{array}{l}\text { Dis- } \\
\text { solved } \\
\text { chloride } \\
\text { (mg/L) }\end{array}$ \\
\hline- & 12 & 140 & -- & -- & -- & -- & -- & -- & -- & -- & 22 \\
\hline-- & 4,500 & 35,000 & -- & 366 & -- & 82.8 & -- & 8.9 & - & 24.4 & 9 \\
\hline- & 7 & 150 & -- & 176 & -- & 36 & -- & 13.4 & -- & 3.3 & 16 \\
\hline -- & 1 & 1,900 & -- & 176 & -- & 38.7 & -- & 12.5 & -- & 3.6 & 20 \\
\hline -- & $<1$ & 90 & -- & 167 & -- & 40.7 & -- & 13.2 & -- & 3.6 & 19 \\
\hline-- & 2 & 250 & -- & 153 & -- & 35.5 & -- & 12.9 & -- & 3.7 & 17 \\
\hline -- & -- & -- & -- & -- & -- & -- & -- & -- & -- & -- & -- \\
\hline -- & $<1$ & 111 & 170 & -- & 37.0 & -- & 13 & -- & 3.5 & -- & 16 \\
\hline 255 & 2 & 20 & -- & 142 & -- & 28.9 & -- & 18.9 & -- & 2.9 & 15 \\
\hline 317 & 4 & $<10$ & 178 & -- & 38.0 & -- & 15.1 & -- & 3.5 & -- & 19 \\
\hline 299 & 2 & $<10$ & 179 & -- & 38.5 & -- & 13.4 & -- & 3.2 & -- & 22.7 \\
\hline -- & $<1$ & $<10$ & 179 & -- & 37.3 & -- & 13.6 & - & 2.9 & -- & 22.5 \\
\hline 270 & 2 & $<10$ & 164 & -- & 38.1 & & 13.6 & $\ldots$ & 2.8 & -- & 23.1 \\
\hline 288 & 2 & $<10$ & 174 & -- & 38.9 & -- & 13.1 & -- & 3.1 & -- & 24.7 \\
\hline 267 & 7 & $<10$ & 151 & -- & 32.7 & -- & 11.4 & -- & 3.1 & -- & 21.3 \\
\hline-- & 7 & 90 & 127 & -- & 28.7 & -- & 9.2 & -- & 2.6 & -- & 16.8 \\
\hline-- & 4 & 2,100 & 169 & -- & 38.3 & -- & 11.7 & -. & 3.1 & -- & 24 \\
\hline 144 & 51 & 1,900 & 56.2 & - & 11.9 & -- & 5.8 & -- & 2.1 & -- & 6 \\
\hline 326 & 2 & 110 & 190 & -- & 40.2 & -- & 13.8 & -- & 4.2 & -- & 26.2 \\
\hline 332 & 4 & 220 & 201 & -- & 43.2 & - & 14.4 & -- & 3.9 & -- & 29.1 \\
\hline-- & $<1$ & 80 & 197 & - & 43.3 & -- & 14.3 & -- & 3.4 & -- & 28.7 \\
\hline 303 & $<1$ & $<10$ & 195 & -- & 41.6 & -- & 15.7 & -- & 3.5 & -- & 26.9 \\
\hline 343 & 2 & 10 & 192 & - & 42.1 & -- & 14.7 & -- & 3.7 & -- & 25.6 \\
\hline 303 & 3 & 12 & 190 & -- & 40.5 & -- & 13.5 & -- & 3.4 & - & 24.3 \\
\hline 327 & 2 & $<2$ & 191 & -- & 40.7 & -- & 14.1 & -- & 3.3 & -- & 26.4 \\
\hline 281 & 3 & 2 & 184 & -- & 40.1 & -- & 14.2 & -- & 2.9 & -- & 26.9 \\
\hline 283 & $<1$ & $<10$ & 190 & -- & 41.5 & -- & 13 & -- & 2.9 & -- & 27.7 \\
\hline 306 & 3 & 10 & 192 & - & 41.3 & -- & 12.9 & -- & 3.1 & -- & 29.5 \\
\hline 268 & 5 & 120 & 181 & -- & 38.6 & -- & 12.4 & -- & 3.1 & -- & 27.3 \\
\hline 262 & 3 & 540 & 137 & -- & 30.7 & -- & 9.8 & -- & 2.5 & -. & 20.4 \\
\hline 260 & 4 & 360 & 140 & -- & 30.5 & -- & 10.1 & -- & 2.4 & -- & 20.6 \\
\hline -- & -- & -- & 137 & -- & 30.5 & -- & 9.7 & -- & 2.4 & -. & -- \\
\hline
\end{tabular}


Table 6. Water-quality data for selected sites along Bear Butte Creek-Continued

$\left[\mathrm{ft}^{3} / \mathrm{s}\right.$, cubic feet per second; $\mu \mathrm{S} / \mathrm{cm}$, microsiemens per centimeter; deg C, degrees Celsius; mg/L, milligrams per liter; $\mu \mathrm{g} / \mathrm{L}$, micrograms per liter; cols, colonies; mL, milliliters; $\mathrm{K}$, non-ideal colony count; <, less than; --, no data available]

\begin{tabular}{|c|c|c|c|c|c|c|c|c|c|c|c|}
\hline Date & Time & $\begin{array}{l}\text { Dis- } \\
\text { solved } \\
\text { sulfate } \\
\text { (mg/L) }\end{array}$ & $\begin{array}{l}\text { Total } \\
\text { sulfate } \\
\text { (mg/L) }\end{array}$ & $\begin{array}{c}\text { Dis- } \\
\text { solved } \\
\text { fluoride } \\
\text { (mg/L) }\end{array}$ & $\begin{array}{c}\text { Total } \\
\text { fluoride } \\
\text { (mg/L) }\end{array}$ & $\begin{array}{l}\text { Nitrate } \\
\text { as nitro- } \\
\text { gen } \\
(\mathrm{mg} / \mathrm{L})\end{array}$ & $\begin{array}{c}\text { Nitrogen } \\
\text { ammonia } \\
\text { (mg/L) }\end{array}$ & $\begin{array}{c}\text { Total } \\
\text { kjeldahl } \\
\text { nitrogen } \\
\text { (mg/L) }\end{array}$ & $\begin{array}{c}\text { Total } \\
\text { dissolved } \\
\text { phos- } \\
\text { phorus } \\
\text { (mg/L) }\end{array}$ & $\begin{array}{l}\text { Dis- } \\
\text { solved } \\
\text { cyanide } \\
(\mathrm{mg} / \mathrm{L})\end{array}$ & $\begin{array}{c}\text { Dis- } \\
\text { sovled } \\
\text { arsenic } \\
(\mu \mathrm{g} / \mathrm{L})\end{array}$ \\
\hline \multicolumn{12}{|c|}{ Site 1 - 06437400 Bear Butte Creek at Sturgis, SD-Continued } \\
\hline 06-16-1998 & 0930 & -- & 360 & -- & 0.26 & 2.6 & $<0.02$ & $<0.1$ & 0.022 & $<0.01$ & -- \\
\hline 06-18-1998 & 1300 & -- & 48.7 & - & .28 & .4 & $<.02$ & 2.87 & .19 & $<.01$ & 3 \\
\hline 07-07-1998 & 0915 & -- & 356 & -- & .24 & 2.8 & $<.02$ & .29 & .008 & $<.01$ & 2.3 \\
\hline $08-11-1998$ & 1015 & -- & 391 & -- & .25 & 2.5 & $<.02$ & .46 & .018 & $<.01$ & 4.1 \\
\hline 09-16-1998 & 0855 & -- & 387 & -- & .23 & 2.5 & $<.02$ & .19 & .004 & $<.01$ & 2.3 \\
\hline $10-06-1998$ & 1040 & -- & 331 & -- & .23 & 2.4 & $<.02$ & .26 & .097 & $<.01$ & 2.6 \\
\hline${ }^{1} 10-06-1998$ & 1041 & -- & -- & -- & -- & -- & -- & -- & -- & -- & 4 \\
\hline${ }^{2} 10-06-1998$ & 1048 & 320 & - & 0.17 & & 2.74 & .021 & .12 & $<.05$ & -- & 2 \\
\hline $11-17-1998$ & 0930 & -- & 280 & -- & .32 & 2.1 & $<.02$ & .26 & .013 & $<.01$ & 2 \\
\hline $12-15-1998$ & 0930 & 340 & - & - & -- & 3.1 & $<.02$ & .37 & .007 & $<.01$ & 2.9 \\
\hline 01-25-1999 & 1125 & 346 & -- & .25 & -- & 3 & $<.02$ & $<.1$ & .012 & $<.01$ & 2.2 \\
\hline 02-10-1999 & 0915 & 356 & - & .24 & -- & 2.9 & $<.02$ & .28 & .007 & $<.01$ & 1.9 \\
\hline 03-09-1999 & 0915 & 344 & - & .25 & -- & 2.8 & $<.02$ & .18 & .005 & $<.01$ & 1.7 \\
\hline 04-06-1999 & 0900 & 330 & - & .26 & -- & 2.6 & $<.02$ & .24 & .004 & $<.01$ & 2.4 \\
\hline 04-21-1999 & 1040 & 287 & -- & .22 & -- & 2.2 & $<.02$ & .34 & .01 & $<.01$ & 2.1 \\
\hline 05-10-1999 & 0900 & 243 & -- & .19 & -- & 2.1 & $<.02$ & .26 & .072 & $<.01$ & .8 \\
\hline 06-09-1999 & 0915 & 333 & - & -- & -- & 2.7 & $<.02$ & .19 & .018 & $<.01$ & 1.2 \\
\hline $06-15-1999$ & 0755 & 71.1 & -- & -- & -- & .6 & $<.02$ & .56 & .154 & $<.01$ & 2.7 \\
\hline 07-19-1999 & 0735 & 374 & - & -- & -- & 3.9 & $<.02$ & .19 & .013 & $<.01$ & 2.6 \\
\hline 08-16-1999 & 0840 & 358 & - & -- & -- & 3.6 & $<.02$ & .25 & .006 & $<.01$ & 2 \\
\hline 09-14-1999 & 0910 & 377 & - & .17 & -- & 3.4 & $<.02$ & .18 & .005 & $<.01$ & 1.5 \\
\hline $10-26-1999$ & 0910 & 359 & - & .24 & - & 3.3 & $<.02$ & $<.14$ & .002 & $<.01$ & 1.4 \\
\hline $11-16-1999$ & 0850 & 359 & - & .38 & - & 3.4 & $<.02$ & .15 & .002 & $<.01$ & 1.3 \\
\hline $12-07-1999$ & 0900 & 360 & -- & .24 & -- & 3.4 & $<.02$ & .2 & .008 & $<.01$ & 1.9 \\
\hline $01-18-2000$ & 0905 & 360 & - & .25 & - & 3.2 & $<.02$ & $<.2$ & .006 & $<.01$ & 1.5 \\
\hline $02-22-2000$ & 0845 & 363 & -- & .24 & -- & 2.9 & $<.02$ & $<.21$ & .008 & $<.01$ & 1.6 \\
\hline $03-28-2000$ & 0900 & 359 & - & .26 & -- & 2.8 & $<.02$ & $<.21$ & .005 & $<.01$ & 1.5 \\
\hline $04-04-2000$ & 0855 & 356 & -- & .25 & -- & 2.9 & $<.02$ & .27 & $<.002$ & $<.01$ & 1.5 \\
\hline $05-09-2000$ & 0900 & 321 & - & .25 & -- & 2.8 & $<.02$ & .29 & .009 & $<.01$ & 1.5 \\
\hline $05-18-2000$ & 0900 & 240 & -- & .22 & -- & 2.1 & $<.02$ & .3 & .015 & $<.01$ & 1.5 \\
\hline${ }^{3} 05-18-2000$ & 0905 & 243 & - & .23 & -- & 2.2 & $<.02$ & .28 & .014 & $<.01$ & 1.3 \\
\hline${ }^{3} 05-18-2000$ & 0908 & - & -- & -- & -- & -- & -- & - & - & $<.01$ & 1.4 \\
\hline
\end{tabular}




\begin{tabular}{|c|c|c|c|c|c|c|c|c|c|c|c|}
\hline $\begin{array}{c}\text { Dis- } \\
\text { solved } \\
\text { cadmium } \\
(\mu \mathrm{g} / \mathrm{L})\end{array}$ & $\begin{array}{l}\text { Dis- } \\
\text { solved } \\
\text { chro- } \\
\text { mium } \\
(\mu \mathrm{g} / \mathrm{L})\end{array}$ & $\begin{array}{c}\text { Dis- } \\
\text { solved } \\
\text { copper } \\
(\mu \mathrm{g} / \mathrm{L})\end{array}$ & $\begin{array}{l}\text { Dis- } \\
\text { solved } \\
\text { iron } \\
(\mathrm{mg} / \mathrm{L})\end{array}$ & $\begin{array}{c}\text { Total } \\
\text { iron } \\
\text { (mg/L) }\end{array}$ & $\begin{array}{c}\text { Dis- } \\
\text { solved } \\
\text { lead } \\
(\mu \mathrm{g} / \mathrm{L})\end{array}$ & $\begin{array}{c}\text { Dis- } \\
\text { solved } \\
\text { manga- } \\
\text { nese } \\
(\mathrm{mg} / \mathrm{L})\end{array}$ & $\begin{array}{c}\text { Total } \\
\text { manga- } \\
\text { neses } \\
(\mathrm{mg} / \mathrm{L})\end{array}$ & $\begin{array}{c}\text { Dis- } \\
\text { solved } \\
\text { mercury } \\
(\mu \mathrm{g} / \mathrm{L})\end{array}$ & $\begin{array}{c}\text { Dis- } \\
\text { solved } \\
\text { nickel } \\
(\mu \mathrm{g} / \mathrm{L})\end{array}$ & $\begin{array}{c}\text { Dis- } \\
\text { solved } \\
\text { silver } \\
(\mu \mathrm{g} / \mathrm{L})\end{array}$ & $\begin{array}{c}\text { Dis- } \\
\text { solved } \\
\text { zinc } \\
(\mu \mathrm{g} / \mathrm{L})\end{array}$ \\
\hline-- & -- & -- & -- & 0.13 & -- & -- & $<0.02$ & -- & -- & -- & -- \\
\hline$<0.5$ & 11.6 & $<0.02$ & -- & 71 & $<0.8$ & -- & 2.96 & $<0.2$ & $<0.9$ & $<0.5$ & $<50$ \\
\hline$<.1$ & 3.3 & $<.02$ & -- & $<.06$ & $<.8$ & -- & $<.02$ & $<.13$ & 4.2 & $<.2$ & $<50$ \\
\hline$<.1$ & 6.8 & $<.02$ & -- & $<.06$ & $<.8$ & -- & $<.02$ & $<.2$ & 5.3 & $<.2$ & 3.7 \\
\hline$<.1$ & 8 & $<.02$ & -- & $<.06$ & $<1$ & -- & .02 & $<.2$ & 4.4 & $<.2$ & 4.3 \\
\hline$<.1$ & 4.6 & $<.02$ & -- & $<.06$ & $<1$ & -- & $<.02$ & $<.2$ & -- & $<1$ & $<3$ \\
\hline$<.1$ & 17.6 & $<.02$ & -- & -- & $<1$ & -- & -- & $<.2$ & 4.1 & $<1$ & 15.2 \\
\hline$<1$ & 2 & .0016 & $<0.01$ & -- & $<1$ & 0.0042 & & $<.1$ & 1.9 & $<1$ & 5.2 \\
\hline$<.2$ & $<.2$ & $<.02$ & -- & $<.06$ & $<.8$ & -- & $<.02$ & $<.2$ & 3.3 & $<.2$ & 6.1 \\
\hline$<.2$ & 3.8 & $<.02$ & $<.02$ & -- & $<.1$ & .02 & - & $<.1$ & 5 & $<.2$ & 4 \\
\hline$<.2$ & 7 & $<.02$ & $<.02$ & -- & $<.8$ & .02 & -- & $<.1$ & 5 & $<.2$ & $<40$ \\
\hline$<.2$ & 4.7 & .7 & .02 & -- & $<.1$ & $<.02$ & -- & $<.1$ & 6.5 & $<.2$ & 5 \\
\hline$<.2$ & 4.1 & .6 & - & - & $<.1$ & -- & -- & $<.1$ & 3.8 & $<.2$ & 3 \\
\hline$<.2$ & 8.6 & .5 & -- & - & $<.1$ & - & - & $<.1$ & 4.3 & $<.2$ & $<3$ \\
\hline$<.2$ & 6 & 1 & -- & -- & $<.1$ & -- & - & $<.1$ & 5 & $<.2$ & 7 \\
\hline$<.2$ & 6.7 & .8 & .03 & -- & $<.1$ & $<.02$ & -- & $<.1$ & 4 & $<.2$ & 4 \\
\hline$<.2$ & 6.7 & $<.02$ & $<.02$ & -- & $<.1$ & .02 & -- & $<.1$ & 4.3 & $<.2$ & 5 \\
\hline$<.2$ & 3.2 & 1.4 & -- & -- & $<.1$ & -- & - & $<.1$ & 2.8 & $<.2$ & 6 \\
\hline$<.2$ & 7.1 & 1.2 & -- & -- & $<.1$ & -- & -- & $<.1$ & 4.4 & $<.2$ & 4 \\
\hline$<.2$ & 10 & .9 & $<.02$ & -- & $<.1$ & .02 & - & $<.1$ & 4.5 & $<.2$ & 4.6 \\
\hline$<.2$ & 6.2 & 1 & $<.02$ & -- & .1 & $<.02$ & - & $<.1$ & 5.5 & $<.2$ & 5.8 \\
\hline .4 & 5.6 & .8 & $<.02$ & -- & $<.1$ & $<.02$ & -- & $<.1$ & 4.8 & $<.2$ & 5 \\
\hline$<.2$ & 3.6 & .6 & $<.02$ & -- & $<.1$ & $<.02$ & -- & $<.1$ & 4.7 & $<.2$ & 5 \\
\hline$<.2$ & 7.9 & .7 & $<.02$ & -- & $<.1$ & $<.02$ & -- & $<.1$ & 4.5 & $<.2$ & 4 \\
\hline$<.2$ & 4.7 & .6 & .02 & -- & $<.1$ & $<.02$ & -- & $<.1$ & 5 & $<.2$ & 4 \\
\hline$<.2$ & 6.6 & .6 & $<.02$ & -- & $<.1$ & $<.02$ & -- & $<.1$ & 5.4 & $<.2$ & 4 \\
\hline$<.2$ & 5.8 & .9 & $<.02$ & -- & $<.1$ & .0031 & -- & $<.1$ & 5.2 & $<.2$ & $<3$ \\
\hline$<.2$ & 8.3 & .7 & $<.02$ & -- & $<.1$ & .0033 & -- & $<.1$ & 5.3 & $<.2$ & $<3$ \\
\hline$<.2$ & 10.5 & .7 & $<.02$ & -- & .1 & $<.02$ & - & $<.1$ & 5.1 & .4 & 4.1 \\
\hline$<.2$ & 9.8 & .6 & $<.02$ & -- & $<.1$ & $<.02$ & -- & $<.1$ & 3.9 & $<.2$ & 3.3 \\
\hline$<.2$ & 11.7 & .6 & .02 & -- & $<.1$ & .02 & -- & $<.1$ & 3.6 & $<.2$ & $<3$ \\
\hline$<.2$ & 11.9 & .6 & .02 & - & $<.1$ & .02 & - & $<.1$ & 3.6 & $<.2$ & 3.3 \\
\hline
\end{tabular}


Table 6. Water-quality data for selected sites along Bear Butte Creek-Continued

$\left[\mathrm{ft}^{3} / \mathrm{s}\right.$, cubic feet per second; $\mu \mathrm{S} / \mathrm{cm}$, microsiemens per centimeter; deg C, degrees Celsius; mg/L, milligrams per liter; $\mu \mathrm{g} / \mathrm{L}$, micrograms per liter; cols, colonies; $\mathrm{mL}$, milliliters; $\mathrm{K}$, non-ideal colony count; <, less than; --, no data available]

\begin{tabular}{|c|c|c|c|c|c|c|c|c|c|c|c|}
\hline Date & Time & $\begin{array}{c}\text { Dis- } \\
\text { charge } \\
\left(\mathrm{ft}^{3} / \mathrm{s}\right)\end{array}$ & $\begin{array}{c}\text { Specific } \\
\text { conduc- } \\
\text { tance } \\
(\mu \mathrm{S} / \mathrm{cm})\end{array}$ & $\begin{array}{c}\mathrm{pH} \\
\text { (standard } \\
\text { units) }\end{array}$ & $\begin{array}{c}\text { Water } \\
\text { tempera- } \\
\text { ture } \\
\text { (deg C) }\end{array}$ & $\begin{array}{c}\text { Air } \\
\text { tempera- } \\
\text { ture } \\
\text { (deg C) }\end{array}$ & $\begin{array}{c}\text { Dis- } \\
\text { solved } \\
\text { oxygen } \\
\text { (mg/L) }\end{array}$ & $\begin{array}{c}\text { Bio- } \\
\text { logical } \\
\text { oxygen } \\
\text { demand } \\
\text { (mg/L) }\end{array}$ & $\begin{array}{c}\text { Carbon } \\
\text { oxygen } \\
\text { demand } \\
(\mathrm{mg} / \mathrm{L})\end{array}$ & $\begin{array}{c}\text { Hardness } \\
\text { as } \mathrm{CaCO}_{3} \\
(\mathrm{mg} / \mathrm{L})\end{array}$ & $\begin{array}{l}\text { Labora- } \\
\text { tory } \\
\text { alkalinity } \\
\text { (mg/L) }\end{array}$ \\
\hline \multicolumn{12}{|c|}{ Site 2 - 442811103205000 Bear Butte Creek below Sturgis, SD } \\
\hline $06-16-1998$ & 1145 & 4.2 & 1,440 & 7.8 & 16.5 & 20.5 & 9.0 & $<1$ & 0.54 & 713 & 252 \\
\hline 06-18-1998 & 1405 & 52 & 1,140 & 7.9 & 12.5 & 13.0 & 8.5 & 8 & 14.8 & 655 & 264 \\
\hline 07-07-1998 & 1100 & 6.2 & 1,370 & 7.9 & 21.5 & 25.0 & 8.3 & $<1$ & 8 & 662 & 239 \\
\hline 08-11-1998 & 1215 & 3.8 & 1,410 & 8.0 & 21.0 & 22.5 & 8.4 & $<1$ & 18.8 & 674 & 236 \\
\hline 09-16-1998 & 1100 & 1.1 & 1,670 & 7.9 & 17.5 & 24.0 & 8.2 & $<3$ & 22.6 & 820 & 246 \\
\hline${ }^{1} 10-06-1998$ & 1345 & -- & -- & -- & -- & -- & 11.2 & -- & -- & -- & -- \\
\hline $10-06-1998$ & 1346 & 17 & 751 & 7.9 & 7.0 & 11.0 & 11.2 & $<1$ & 14.1 & 355 & 151 \\
\hline${ }^{2} 10-06-1998$ & 1350 & 17 & 751 & 7.9 & 7.0 & 11.0 & 11.2 & - & -- & -- & 146 \\
\hline $11-17-1998$ & 1130 & 22 & 1,250 & 7.9 & 5.5 & 6.0 & 13.3 & $<1$ & $<20$ & 629 & 231 \\
\hline $12-15-1998$ & 1115 & 10 & 1,360 & 7.6 & 2.0 & 8.0 & 15.2 & $<1$ & $<20$ & 679 & 249 \\
\hline 01-25-1999 & 1300 & 8.4 & 1,580 & 7.8 & .0 & 9.0 & 12.4 & $<1$ & $<10$ & 800 & 279 \\
\hline 02-10-1999 & 1055 & 6.6 & 1,490 & 7.9 & 3.0 & 7.0 & 14.5 & $<1$ & $<10$ & 728 & 269 \\
\hline 03-09-1999 & 1105 & 6.3 & 1,530 & 7.7 & 1.5 & 5.0 & 16.3 & $<1$ & $<10$ & 750 & 247 \\
\hline 04-06-1999 & 1055 & 20 & 1,730 & 8.0 & 5.5 & 6.0 & 11.2 & $<2$ & 14.8 & 881 & 278 \\
\hline 04-21-1999 & 1144 & 15 & 1,530 & 7.8 & 10.0 & 7.5 & 10.1 & $<1$ & $<10$ & 748 & 252 \\
\hline 05-10-1999 & 1130 & 16 & 1,470 & 7.9 & 8.5 & 4.0 & 5.4 & $<1$ & 17.9 & 732 & 254 \\
\hline 06-09-1999 & 1100 & 17 & 1,730 & 8.1 & 16.5 & 18.0 & 8.2 & $<1$ & 11.8 & 801 & 274 \\
\hline 06-15-1999 & 0940 & 123 & 755 & 8.0 & 13.5 & 15.0 & 7.8 & $<2$ & $<10$ & 349 & 179 \\
\hline 07-19-1999 & 0925 & 14 & 1,380 & 7.7 & 19.5 & 26.0 & 6.9 & $<1$ & 10.8 & 694 & 263 \\
\hline 08-16-1999 & 1025 & 6.8 & 1,430 & 7.7 & 18.5 & 19.5 & 8.2 & $<1$ & $<10$ & 742 & 247 \\
\hline 09-14-1999 & 1100 & 5.2 & 1,470 & 7.5 & 11.0 & 12.0 & 11.3 & $<1$ & $<10$ & 744 & 231 \\
\hline $10-26-1999$ & 1050 & 5.6 & 1,530 & 7.7 & 8.0 & 15.0 & 10.9 & $<1$ & $<10$ & 772 & 252 \\
\hline $11-16-1999$ & 1025 & 5.7 & 1,510 & 7.7 & 5.5 & 6.0 & 11.7 & $<1$ & $<10$ & 775 & 266 \\
\hline $12-07-1999$ & 1050 & 5.5 & 1,570 & 7.4 & 1.0 & 4.0 & 14.0 & $<1$ & $<10$ & 778 & 249 \\
\hline $01-18-2000$ & 1105 & 4.6 & 1,660 & 7.9 & .0 & .0 & 12.1 & $<1$ & $<10$ & 822 & 275 \\
\hline $02-22-2000$ & 1030 & 4.5 & 1,610 & 7.9 & 1.5 & 12.0 & 12.7 & $<1$ & $<10$ & 774 & 234 \\
\hline $03-28-2000$ & 1130 & 7.5 & 1,650 & 7.9 & 6.5 & 12.0 & 10.6 & $<2$ & $<10$ & 839 & 235 \\
\hline${ }^{2} 03-28-2000$ & 1135 & 7.5 & 1,650 & 7.9 & 6.5 & 12.0 & 10.6 & -- & -- & 800 & 259 \\
\hline 04-04-2000 & 1035 & 6.3 & 1,620 & 7.9 & 4.5 & 10.0 & 11.0 & $<1$ & $<10$ & 811 & 256 \\
\hline $05-09-2000$ & 1030 & 16 & 1,460 & 8.2 & 11.0 & 15.5 & 11.3 & $<1$ & $<10$ & 743 & 236 \\
\hline $05-18-2000$ & 1100 & 19 & 1,440 & 8.3 & 10.0 & 9.0 & 10.5 & $<1$ & 24.1 & 733 & 255 \\
\hline
\end{tabular}




\begin{tabular}{|c|c|c|c|c|c|c|c|c|c|c|c|}
\hline $\begin{array}{c}\text { Bicar- } \\
\text { bonate as } \\
\mathrm{CaCO}_{3} \\
(\mathrm{mg} / \mathrm{L})\end{array}$ & $\begin{array}{c}\text { Total } \\
\text { sus- } \\
\text { pended } \\
\text { solids } \\
\text { (mg/L) }\end{array}$ & $\begin{array}{c}\text { Fecal } \\
\text { coliform } \\
\text { (cols/ } \\
100 \mathrm{~mL} \text { ) }\end{array}$ & $\begin{array}{c}\text { Dis- } \\
\text { solved } \\
\text { calcium } \\
\text { (mg/L) }\end{array}$ & $\begin{array}{l}\text { Total } \\
\text { calcium } \\
\text { (mg/L) }\end{array}$ & $\begin{array}{c}\text { Dis- } \\
\text { solved } \\
\text { magne- } \\
\text { sium } \\
(\mathrm{mg} / \mathrm{L})\end{array}$ & $\begin{array}{l}\text { Total } \\
\text { magne- } \\
\text { sium } \\
(\mathrm{mg} / \mathrm{L})\end{array}$ & $\begin{array}{l}\text { Dis- } \\
\text { solved } \\
\text { sodium } \\
\text { (mg/L) }\end{array}$ & $\begin{array}{c}\text { Total } \\
\text { sodium } \\
\text { (mg/L) }\end{array}$ & $\begin{array}{l}\text { Dis- } \\
\text { solved } \\
\text { potas- } \\
\text { sium } \\
(\mathrm{mg} / \mathrm{L})\end{array}$ & $\begin{array}{l}\text { Total } \\
\text { potas- } \\
\text { sium } \\
(\mathrm{mg} / \mathrm{L})\end{array}$ & $\begin{array}{c}\text { Dis- } \\
\text { solved } \\
\text { chloride } \\
\text { (mg/L) }\end{array}$ \\
\hline-- & 40 & 120 & -- & 183 & -- & 62.3 & -- & 46.9 & -- & 5.6 & 40 \\
\hline -- & 652 & 17,000 & -- & 183 & -- & 48.2 & -- & 35.6 & -- & 19.5 & 22 \\
\hline-- & 38 & 480 & -- & 173 & -- & 56 & -- & 44.4 & -- & 5.5 & 32 \\
\hline- & 30 & 770 & -- & 170 & -- & 60.8 & -- & 47.8 & -- & 6.2 & 37 \\
\hline-- & 18 & 350 & -- & 191 & -- & 78.9 & -- & 63.4 & -- & 7.8 & 45 \\
\hline-- & -- & - & -- & -- & -- & - & -- & -- & -- & -- & -- \\
\hline-- & 52 & 3,200 & -- & 100 & -- & 25.5 & -- & 16.4 & -- & 4.9 & 20 \\
\hline- & 47 & K2,840 & 95 & -- & 26 & -- & 18 & -- & 4.6 & -- & 18 \\
\hline 282 & 10 & 170 & -- & 170 & - & 49.7 & -- & 40.8 & -- & 5.2 & 26 \\
\hline 304 & 5 & 40 & 177 & -- & 57.6 & -- & 43.6 & - & 5.1 & -- & 31 \\
\hline-- & 3 & 30 & 203 & -- & 66.3 & -- & 49.6 & - & 5.3 & -- & 38.5 \\
\hline 328 & 6 & 40 & 188 & - & 62.9 & - & 51.3 & -- & 5 & -- & 36.9 \\
\hline 301 & 3 & 10 & 182 & -- & 66.6 & -- & 52.9 & -- & 4.9 & -- & 37.6 \\
\hline 339 & 32 & 540 & 221 & -- & 80 & - & 198 & -- & 6.5 & -- & 41.7 \\
\hline 307 & 17 & $<10$ & 191 & -- & 65.8 & -- & 55.9 & - & 5.4 & -- & 34.4 \\
\hline -- & 17 & 1,400 & 186 & -- & 64.9 & -- & 51.4 & -- & 5.3 & -- & 33.3 \\
\hline-- & 46 & 4,300 & 192 & -- & 78.1 & -- & 94.5 & -- & 5.9 & -- & 29.7 \\
\hline 218 & 148 & 2,100 & 96.4 & -- & 26.2 & - & 22.2 & -- & 3.4 & -- & 10.9 \\
\hline 321 & 21 & 430 & 185 & -- & 56.3 & - & 41.7 & - & 6 & -- & 33.2 \\
\hline 301 & 25 & 380 & 192 & -- & 63.9 & -- & 46.4 & -- & 6.1 & - & 37.2 \\
\hline -- & 16 & 190 & 191 & -- & 64.8 & -- & 48.4 & -- & 5.9 & -- & 38.3 \\
\hline 307 & 11 & 10 & 199 & - & 66.9 & -- & 55.2 & -- & 5.4 & -- & 41 \\
\hline 325 & 7 & 50 & 200 & -- & 67 & -- & 51.7 & - & 6 & -- & 41.1 \\
\hline 304 & 6 & 100 & 204 & - & 65.2 & - & 50.5 & -- & 5.5 & -- & 41.4 \\
\hline 336 & 1 & 4 & 214 & -- & 69.8 & -- & 56.1 & -- & 5.5 & - & 43.3 \\
\hline 285 & 5 & 10 & 199 & -- & 67.4 & -- & 54.8 & -- & 5 & -- & 41.2 \\
\hline 287 & 14 & 240 & 216 & -- & 72.7 & -- & 56.7 & -- & 5.4 & - & 40.7 \\
\hline-- & 13 & -- & 203.7 & -- & 79.3 & -- & 59.5 & -- & 5.76 & -- & 39.9 \\
\hline 312 & 9 & 1,100 & 210 & -- & 69.7 & -- & 57.5 & -- & 5.2 & -- & 42.2 \\
\hline 288 & 15 & 250 & 193 & -- & 63.4 & -- & 53.7 & -- & 5.3 & -- & 31.9 \\
\hline 311 & 33 & 650 & 192 & -- & 61.7 & -- & 52 & -- & 4.7 & -- & 36 \\
\hline
\end{tabular}


Table 6. Water-quality data for selected sites along Bear Butte Creek-Continued

$\left[\mathrm{ft}^{3} / \mathrm{s}\right.$, cubic feet per second; $\mu \mathrm{S} / \mathrm{cm}$, microsiemens per centimeter; deg C, degrees Celsius; mg/L, milligrams per liter; $\mu \mathrm{g} / \mathrm{L}$, micrograms per liter; cols, colonies; mL, milliliters; K. non-ideal colony count; $<$, less than: --, no data available]

\begin{tabular}{|c|c|c|c|c|c|c|c|c|c|c|c|}
\hline Date & Time & $\begin{array}{l}\text { Dis- } \\
\text { solved } \\
\text { sulfate } \\
\text { (mg/L) }\end{array}$ & $\begin{array}{l}\text { Total } \\
\text { sulfate } \\
\text { (mg/L) }\end{array}$ & $\begin{array}{c}\text { Dis- } \\
\text { solved } \\
\text { fluoride } \\
\text { (mg/L) }\end{array}$ & $\begin{array}{l}\text { Total } \\
\text { fluoride } \\
\text { (mg/L) }\end{array}$ & $\begin{array}{l}\text { Nitrate } \\
\text { as nitro- } \\
\text { gen } \\
(\mathrm{mg} / \mathrm{L})\end{array}$ & $\begin{array}{c}\text { Nitrogen } \\
\text { ammonia } \\
(\mathrm{mg} / \mathrm{L})\end{array}$ & $\begin{array}{c}\text { Total } \\
\text { kjeldahl } \\
\text { nitrogen } \\
\text { (mg/L) }\end{array}$ & $\begin{array}{c}\text { Total } \\
\text { dissolved } \\
\text { phos- } \\
\text { phorus } \\
\text { (mg/L) }\end{array}$ & $\begin{array}{c}\text { Dis- } \\
\text { solved } \\
\text { cyanide } \\
(\mathrm{mg} / \mathrm{L})\end{array}$ & $\begin{array}{c}\text { Dis- } \\
\text { sovled } \\
\text { arsenic } \\
(\mu \mathrm{g} / L)\end{array}$ \\
\hline \multicolumn{12}{|c|}{ Site 2 - 442811103205000 Bear Butte Creek below Sturgis, SD—Continued } \\
\hline 06-16-1998 & 1145 & - & 527 & -- & 0.32 & 0.4 & $<0.02$ & 0.3 & 0.075 & $<0.01$ & 3.9 \\
\hline 06-18-1998 & 1405 & -- & 370 & -- & .31 & .3 & $<.02$ & 1.24 & .8 & $<.01$ & 4.4 \\
\hline 07-07-1998 & 1100 & -- & 489 & -- & .31 & .4 & $<.02$ & .36 & .069 & $<.01$ & 3.5 \\
\hline 08-11-1998 & 1215 & - & 546 & -- & .31 & .2 & $<.02$ & .67 & .071 & $<.01$ & 5.2 \\
\hline $09-16-1998$ & 1100 & -- & 656 & -- & .34 & $<.1$ & $<.02$ & .6 & .052 & $<.01$ & 2.8 \\
\hline${ }^{1} 10-06-1998$ & 1345 & -- & -- & - & -- & -- & -- & - & -- & - & 3.7 \\
\hline $10-06-1998$ & 1346 & -- & 221 & -- & .18 & 6 & $<.02$ & .63 & .108 & $<.01$ & 3 \\
\hline${ }^{2} 10-06-1998$ & 1350 & 210 & -- & 0.11 & -- & .603 & .025 & .18 & .068 & -- & 2 \\
\hline $11-17-1998$ & 1130 & -- & 431 & -- & .26 & .7 & $<.02$ & .38 & .023 & $<.01$ & 1.7 \\
\hline $12-15-1998$ & 1115 & 493 & -- & -- & -- & .9 & $<.02$ & .38 & .009 & $<.01$ & 2.6 \\
\hline $01-25-1999$ & 1300 & 548 & -- & .34 & -- & .9 & $<.02$ & .12 & .015 & $<.01$ & 1.9 \\
\hline 02-10-1999 & 1055 & 539 & -- & .3 & -- & .7 & $<.02$ & .41 & .019 & $<.01$ & 1.3 \\
\hline 03-09-1999 & 1105 & 563 & -- & .3 & -- & .7 & $<.02$ & .39 & .017 & $<.01$ & 2.1 \\
\hline 04-06-1999 & 1055 & 938 & -- & .37 & -- & .4 & $<.02$ & .48 & .045 & $<.01$ & 2.2 \\
\hline 04-21-1999 & 1144 & 551 & - & .32 & -- & 6 & $<.02$ & .47 & .021 & $<.01$ & 2.7 \\
\hline 05-10-1999 & 1130 & 538 & - & .32 & -- & .8 & $<.02$ & .44 & .031 & $<.01$ & 1.6 \\
\hline 06-09-1999 & 1100 & 682 & - & - & -- & .8 & $<.02$ & .71 & .08 & $<.01$ & 2.9 \\
\hline 06-15-1999 & 0940 & 200 & - & -- & -- & 6 & $<.02$ & .83 & .2 & $<.01$ & 3.4 \\
\hline 07-19-1999 & 0925 & 496 & -- & - & -- & 1.3 & $<.02$ & .37 & .042 & $<.01$ & 2.8 \\
\hline 08-16-1999 & 1025 & 519 & -- & -- & -- & .9 & $<.02$ & .5 & .037 & $<.01$ & 2.7 \\
\hline 09-14-1999 & $\$ 100$ & 546 & -- & .2 & -- & .8 & $<.02$ & .29 & .02 & $<.01$ & 1.9 \\
\hline $10-26-1999$ & 1050 & 550 & -- & .26 & -- & .6 & $<.02$ & .27 & .021 & $<.01$ & 1.8 \\
\hline 11-16-1999 & 1025 & 567 & -- & .31 & -- & .7 & $<.02$ & .26 & .014 & $<.01$ & 1.3 \\
\hline $12-07-1999$ & 1050 & 562 & -- & .29 & -- & .9 & $<.02$ & .24 & .009 & $<.01$ & 1.6 \\
\hline $01-18-2000$ & 1105 & 586 & -- & .31 & -- & .9 & $<.02$ & .32 & .006 & $<.01$ & 1.2 \\
\hline $02-22-2000$ & 1030 & 583 & - & .29 & -- & .7 & $<.02$ & .33 & .01 & $<.01$ & 1.1 \\
\hline $03-28-2000$ & 1130 & 646 & -- & .32 & -- & .5 & $<.02$ & .45 & .032 & $<.01$ & 1.5 \\
\hline${ }^{2} 03-28-2000$ & 1135 & 652 & -- & .25 & -- & .488 & $<.02$ & .3 & .05 & $<.01$ & 1.6 \\
\hline $04-04-2000$ & 1035 & 615 & -- & .29 & -. & .5 & $<.02$ & .44 & .02 & $<.01$ & 1.5 \\
\hline $05-09-2000$ & 1030 & 560 & -- & .32 & -- & .7 & $<.02$ & .48 & .031 & $<.01$ & 2 \\
\hline $05-18-2000$ & 1100 & 535 & -- & .29 & -- & .7 & $<.02$ & .54 & .037 & $<.01$ & 1.9 \\
\hline
\end{tabular}




\begin{tabular}{|c|c|c|c|c|c|c|c|c|c|c|c|}
\hline $\begin{array}{c}\text { Dis- } \\
\text { solved } \\
\text { cadmium } \\
(\mu \mathrm{g} / \mathrm{L})\end{array}$ & $\begin{array}{l}\text { Dis- } \\
\text { solved } \\
\text { chro- } \\
\text { mium } \\
(\mu \mathrm{g} / \mathrm{L})\end{array}$ & $\begin{array}{c}\text { Dis- } \\
\text { solved } \\
\text { copper } \\
(\mu \mathrm{g} / \mathrm{L})\end{array}$ & $\begin{array}{l}\text { Dis- } \\
\text { solved } \\
\text { iron } \\
(\mathrm{mg} / \mathrm{L})\end{array}$ & $\begin{array}{c}\text { Total } \\
\text { iron } \\
\text { (mg/L) }\end{array}$ & $\begin{array}{c}\text { Dis- } \\
\text { solved } \\
\text { lead } \\
(\mu \mathrm{g} / \mathrm{L})\end{array}$ & $\begin{array}{c}\text { Dis- } \\
\text { solved } \\
\text { manga- } \\
\text { nese } \\
(\mathrm{mg} / \mathrm{L})\end{array}$ & $\begin{array}{c}\text { Total } \\
\text { manga- } \\
\text { neses } \\
(\mathrm{mg} / \mathrm{L})\end{array}$ & $\begin{array}{c}\text { Dis- } \\
\text { solved } \\
\text { mercury } \\
(\mu g / L)\end{array}$ & $\begin{array}{c}\text { Dis- } \\
\text { solved } \\
\text { nickel } \\
(\mu \mathrm{g} / \mathrm{L})\end{array}$ & $\begin{array}{l}\text { Dis- } \\
\text { solved } \\
\text { silver } \\
(\mu \mathrm{g} / \mathrm{L})\end{array}$ & $\begin{array}{c}\text { Dis- } \\
\text { solved } \\
\text { zinc } \\
(\mu \mathrm{g} / \mathrm{L})\end{array}$ \\
\hline$<0.5$ & 9.4 & $<0.02$ & -- & 0.84 & $<0.8$ & -- & 0.06 & - & $<0.9$ & $<0.5$ & $<50$ \\
\hline$<.5$ & 5.4 & $<.02$ & -- & 13.3 & $<.8$ & -- & .38 & $<0.2$ & 2.2 & $<.5$ & $<50$ \\
\hline$<.1$ & 7.9 & $<.02$ & -- & 1 & $<.8$ & -- & .08 & $<.13$ & 5 & $<.2$ & $<50$ \\
\hline$<.1$ & 6 & $<.02$ & -- & .36 & $<.8$ & -- & .02 & $<.2$ & 5.5 & $<.2$ & $<3$ \\
\hline$<.1$ & 7.3 & $<.02$ & -- & .33 & $<1$ & -- & .04 & $<.2$ & 5.3 & $<.2$ & $<3$ \\
\hline$<.1$ & 15.3 & $<.02$ & - & -- & $<1$ & -- & - & $<.2$ & $<1$ & $<1$ & $<3$ \\
\hline$<.1$ & $<1$ & $<.2$ & -- & 1.08 & $<1$ & -- & .03 & $<.2$ & $<1$ & $<1$ & $<3$ \\
\hline$<1$ & 1.4 & .0013 & 0.0054 & - & $<1$ & .012 & - & $<.1$ & 1.8 & $<1$ & 1.4 \\
\hline$<.2$ & 4.1 & $<.02$ & -- & $<.06$ & $<.8$ & -- & .04 & $<.2$ & 4.7 & $<.2$ & 2.9 \\
\hline$<.2$ & 3.6 & $<.02$ & -- & -- & .1 & -- & - & $<.1$ & 6.78 & $<.2$ & 3 \\
\hline$<.2$ & 7.5 & $<.02$ & .04 & -- & $<.8$ & .04 & -- & $<.1$ & 6.2 & .4 & $<40$ \\
\hline$<.2$ & 3.4 & 1.2 & .04 & - & .1 & .05 & -- & .1 & 8.2 & $<.2$ & 4 \\
\hline$<.2$ & 5.5 & 1 & -- & -. & $<.1$ & -- & -- & $<.1$ & 4.9 & $<.2$ & $<3$ \\
\hline$<.2$ & 9.4 & 1 & -- & - & $<.1$ & -- & -- & $<.1$ & 7 & $<.2$ & $<3$ \\
\hline$<.2$ & 6.6 & 1.1 & -- & -- & .2 & -- & -- & $<.1$ & 6.6 & $<.2$ & 3 \\
\hline$<.2$ & 7.9 & 1 & .05 & -- & .2 & .05 & - & .1 & 6 & $<.2$ & 3 \\
\hline$<.2$ & $<1.6$ & $<.02$ & .02 & -- & $<.1$ & .04 & -- & $<.1$ & 6.3 & $<.2$ & 4 \\
\hline$<.2$ & 3.7 & 3.5 & -- & -- & $<.1$ & -- & -- & $<.1$ & 5.7 & $<.2$ & 5 \\
\hline$<.2$ & 6.8 & .8 & - & -- & .1 & -- & -- & $<.1$ & 5 & .2 & 3 \\
\hline$<.2$ & 8.7 & $<.9$ & $<.02$ & -- & $<.1$ & .03 & - & $<.1$ & 5.9 & $<.2$ & 3 \\
\hline$<.2$ & 5.2 & .8 & .03 & -- & $<.1$ & .03 & - & $<.1$ & 6.1 & $<.2$ & $<3$ \\
\hline$<.2$ & 5.6 & 1 & .03 & -- & .1 & $<.02$ & - & .2 & 6.4 & .2 & 5 \\
\hline$<.2$ & 3.9 & .7 & .03 & -- & $<.1$ & .02 & -- & $<.1$ & 5.8 & $<.2$ & $<3$ \\
\hline$<.2$ & 7.8 & .7 & .02 & -- & $<.1$ & .02 & -- & $<.1$ & 5.8 & .2 & 3 \\
\hline$<.2$ & 4.9 & 1 & .04 & -- & $<.1$ & .03 & -- & $<.1$ & 6.2 & $<.2$ & 3 \\
\hline$<.2$ & 6.6 & 1 & .02 & -- & $<.1$ & .03 & -- & $<.1$ & 6.3 & $<.2$ & $<3$ \\
\hline$<.2$ & 8.1 & 1 & .03 & -- & $<.1$ & .06 & - & $<.1$ & 7.3 & $<.2$ & $<3$ \\
\hline$<1$ & $<1$ & 1.18 & .021 & -- & $<1$ & .06 & -- & $<.23$ & $<1$ & $<1$ & 2.52 \\
\hline$<.2$ & 8 & 1.1 & .03 & - & $<.1$ & .04 & - & $<.1$ & 7.1 & $<.2$ & $<3$ \\
\hline$<.2$ & 4.4 & 1 & .03 & -- & .1 & .04 & -- & $<.1$ & 6.6 & $<.2$ & $<3$ \\
\hline$<.2$ & 14.3 & .8 & .07 & -- & .2 & .04 & -- & $<.1$ & 6.5 & $<.2$ & $<3$ \\
\hline
\end{tabular}


Table 6. Water-quality data for selected sites along Bear Butte Creek-Continued

$\left[\mathrm{ft}^{3} / \mathrm{s}\right.$, cubic feet per second; $\mu \mathrm{S} / \mathrm{cm}$, microsiemens per centimeter; deg $\mathrm{C}$, degrees Celsius; mg/L, milligrams per liter; $\mu \mathrm{g} / \mathrm{L}$, micrograms per liter; cols, colonies; mL, milliliters: K. non-ideal colony count: <, less than: --, no data available]

\begin{tabular}{|c|c|c|c|c|c|c|c|c|c|c|c|}
\hline Date & Time & $\begin{array}{c}\text { Dis- } \\
\text { charge } \\
\left(\mathrm{ft}^{3} / \mathrm{s}\right)\end{array}$ & $\begin{array}{c}\text { Specific } \\
\text { conduc- } \\
\text { tance } \\
(\mu \mathrm{S} / \mathrm{cm})\end{array}$ & $\begin{array}{c}\text { pH } \\
\text { (standard } \\
\text { units) }\end{array}$ & $\begin{array}{c}\text { Water } \\
\text { tempera- } \\
\text { ture } \\
\text { (deg C) }\end{array}$ & $\begin{array}{c}\text { Air } \\
\text { tempera- } \\
\text { ture } \\
\text { (deg C) }\end{array}$ & $\begin{array}{c}\text { Dis- } \\
\text { solved } \\
\text { oxygen } \\
\text { (mg/L) }\end{array}$ & $\begin{array}{c}\text { Bio- } \\
\text { logical } \\
\text { oxygen } \\
\text { demand } \\
\text { (mg/L) }\end{array}$ & $\begin{array}{c}\text { Carbon } \\
\text { oxygen } \\
\text { demand } \\
\text { (mg/L) }\end{array}$ & $\begin{array}{l}\text { Hardness } \\
\text { as } \mathrm{CaCO}_{3} \\
(\mathrm{mg} / \mathrm{L})\end{array}$ & $\begin{array}{l}\text { Labora- } \\
\text { tory } \\
\text { alkalinity } \\
\text { (mg/L) }\end{array}$ \\
\hline \multicolumn{12}{|c|}{ Site 3 - 06437500 Bear Butte Creek near Sturgis, SD } \\
\hline 06-16-1998 & 1315 & 7.5 & 2,040 & 7.7 & 17.5 & 23.0 & 8.3 & $<1$ & 5.2 & 1,014 & 241 \\
\hline 06-18-1998 & 1532 & 301 & 1,170 & 7.8 & 12.0 & 15.0 & 9.1 & 16 & 16.4 & 862 & 260 \\
\hline 07-07-1998 & 1240 & 12 & 2,080 & 8.0 & 24.0 & 25.0 & 9.2 & $<3$ & 10.2 & 992 & 236 \\
\hline 08-11-1998 & 1340 & 3.5 & 2,050 & 8.1 & 23.5 & 28.5 & 10.2 & $<2$ & 23.9 & 1,078 & 209 \\
\hline 09-16-1998 & 1230 & 1.1 & 2,240 & 8.0 & 19.0 & 26.0 & 8.8 & $<2$ & 32.6 & 980 & 234 \\
\hline $10-07-1998$ & 1035 & 48 & 1,040 & 7.6 & 5.5 & 9.0 & 12.4 & $<2$ & 26.2 & 450 & 105 \\
\hline${ }^{3} 10-07-1998$ & 1036 & 48 & 1,040 & 7.6 & 5.5 & 9.0 & 12.4 & $<3$ & 21.3 & 450 & 105 \\
\hline $11-17-1998$ & 1250 & 51 & 1,710 & 7.9 & 4.0 & 3.5 & 14.1 & $<1$ & $<20$ & 784 & 203 \\
\hline $12-15-1998$ & 1250 & 20 & 2,000 & 7.7 & 1.5 & 4.5 & 16.4 & $<2$ & $<20$ & 1,002 & 251 \\
\hline 01-26-1999 & 0855 & 15 & 2,160 & 7.7 & .0 & -6.5 & 12.1 & $<1$ & 10.5 & 1,100 & 283 \\
\hline 02-10-1999 & 1230 & 18 & 2,090 & 8.0 & 2.5 & 4.1 & 13.8 & $<1$ & 16.8 & 991 & 214 \\
\hline 03-09-1999 & 1245 & 18 & 2,200 & 7.9 & 2.0 & .0 & 14.3 & $<1$ & 10.9 & 1,070 & 238 \\
\hline 04-06-1999 & 1220 & 69 & 2,110 & 8.0 & 6.5 & 8.5 & 10.7 & $<3$ & 25.7 & 914 & 219 \\
\hline 04-21-1999 & 1235 & 29 & 2,140 & 8.1 & 11.0 & 8.0 & 10.6 & $<1$ & 12.9 & 1,011 & 238 \\
\hline 05-10-1999 & 1310 & 32 & 2,020 & 8.0 & 9.0 & 4.0 & 4.9 & $<1$ & 20.4 & 976 & 243 \\
\hline 06-09-1999 & 1245 & 80 & 1,870 & 8.1 & 18.5 & 21.0 & 7.5 & $<3$ & 25.8 & 811 & 214 \\
\hline 06-15-1999 & 1105 & 193 & 1,240 & 7.8 & 16.0 & 16.8 & 7.3 & $<2$ & 18.4 & 556 & 203 \\
\hline 07-19-1999 & 1050 & 30 & 2,000 & 7.8 & 21.5 & 29.5 & 6.0 & $<1$ & 25.4 & 1,007 & 213 \\
\hline 08-16-1999 & 1145 & 12 & 2,090 & 7.9 & 20.0 & 23.0 & 8.7 & $<1$ & 12.4 & 1,080 & 216 \\
\hline 09-14-1999 & 1245 & 9.6 & 2,170 & 7.8 & 12.0 & 16.5 & 10.7 & $<1$ & 14.8 & 1,056 & 225 \\
\hline $10-26-1999$ & 1220 & 10 & 2,240 & 8.0 & 8.0 & 19.5 & 11.7 & $<1$ & 10.8 & 1,100 & 237 \\
\hline 11-16-1999 & 1155 & 10 & 2,180 & 7.9 & 5.0 & 8.5 & 13.5 & $<1$ & 13.4 & 1,091 & 257 \\
\hline $12-07-1999$ & 1205 & 12 & 2,220 & 7.6 & .0 & 6.0 & 14.5 & $<1$ & $<10$ & 1,059 & 243 \\
\hline $01-18-2000$ & 1255 & 9.4 & 2,400 & 8.0 & .0 & 3.0 & 13.0 & $<1$ & 14.7 & 1.163 & 295 \\
\hline $02-22-2000$ & 1200 & 9.4 & 2,210 & 8.1 & 2.0 & 17.0 & 13.6 & $<2$ & $<10$ & 1,030 & 225 \\
\hline $03-28-2000$ & 1400 & 19 & 2,130 & 8.2 & 8.5 & 15.5 & 10.8 & $<2$ & 14.7 & 998 & 232 \\
\hline${ }^{3} 03-28-2000$ & 1402 & 19 & 2,130 & 8.2 & 8.5 & 15.5 & 10.8 & $<2$ & 14.4 & 989 & 227 \\
\hline${ }^{2} 03-28-2000$ & 1405 & 19 & 2,130 & 8.2 & 8.5 & 15.5 & 10.8 & -- & -- & 980 & 234 \\
\hline $04-04-2000$ & 1200 & 14 & 2,200 & 8.0 & 5.5 & 15.0 & 12.2 & $<1$ & 11.5 & 1,070 & 242 \\
\hline $05-09-2000$ & 1215 & 36 & 2,020 & 8.3 & 13.5 & 18.0 & 10.4 & $<2$ & 15.8 & 954 & 236 \\
\hline $05-18-2000$ & 1230 & 33 & 2,010 & 8.3 & 12.0 & 12.0 & 10.4 & $<2$ & 26.4 & 944 & 236 \\
\hline
\end{tabular}




\begin{tabular}{|c|c|c|c|c|c|c|c|c|c|c|c|}
\hline $\begin{array}{c}\text { Bicar- } \\
\text { bonate as } \\
\mathrm{CaCO}_{3} \\
(\mathrm{mg} / \mathrm{L})\end{array}$ & $\begin{array}{l}\text { Total } \\
\text { sus- } \\
\text { pended } \\
\text { solids } \\
\text { (mg/L) }\end{array}$ & $\begin{array}{l}\text { Fecal } \\
\text { coliform } \\
(\text { cols/ } \\
100 \mathrm{~mL})\end{array}$ & $\begin{array}{l}\text { Dis- } \\
\text { solved } \\
\text { calcium } \\
\text { (mg/L) }\end{array}$ & $\begin{array}{l}\text { Total } \\
\text { calcium } \\
\text { (mg/L) }\end{array}$ & $\begin{array}{c}\text { Dis- } \\
\text { solved } \\
\text { magne- } \\
\text { sium } \\
(\mathrm{mg} / \mathrm{L})\end{array}$ & $\begin{array}{c}\text { Total } \\
\text { magne- } \\
\text { sium } \\
(\mathrm{mg} / \mathrm{L})\end{array}$ & $\begin{array}{l}\text { Dis- } \\
\text { solved } \\
\text { sodium } \\
\text { (mg/L) }\end{array}$ & $\begin{array}{c}\text { Total } \\
\text { sodium } \\
\text { (mg/L) }\end{array}$ & $\begin{array}{l}\text { Dis- } \\
\text { solved } \\
\text { potas- } \\
\text { sium } \\
(\mathrm{mg} / \mathrm{L})\end{array}$ & $\begin{array}{c}\text { Total } \\
\text { potas- } \\
\text { sium } \\
(\mathrm{mg} / \mathrm{L})\end{array}$ & $\begin{array}{c}\text { Dis- } \\
\text { solved } \\
\text { chloride } \\
\text { (mg/L) }\end{array}$ \\
\hline- & 79 & 170 & -- & 245 & - & 97.8 & -- & 91.6 & -- & 7.1 & 43 \\
\hline-- & 1,930 & 23,000 & -- & 230 & -- & 69.8 & -- & 98.3 & -- & 18.4 & 33 \\
\hline- & 70 & 430 & -- & 244 & -- & 92.9 & -- & 106 & -- & 8.2 & 34 \\
\hline- & 26 & 130 & - & 238 & -- & 99.4 & -- & 90.9 & - & 6.7 & 40 \\
\hline-- & 14 & -- & -- & 239 & -- & 112 & -- & 102 & -- & 6.3 & 50 \\
\hline- & 200 & 2,300 & - & 115 & -- & 39.5 & -- & 53.1 & -- & 9.4 & 12 \\
\hline- & 196 & 3,600 & -- & 114 & -- & 40.1 & -- & 53.7 & -- & 9.5 & 13 \\
\hline 248 & 24 & 50 & -- & 187 & -- & 77 & -- & 102 & -- & 8.2 & 24 \\
\hline 306 & 10 & 60 & 247 & -- & 93.5 & -- & 98.7 & - & 6.5 & - & 32 \\
\hline-- & 4 & 230 & 271 & - & 96.9 & -- & 98.7 & -- & 6.5 & -- & 36.5 \\
\hline 261 & 9 & 100 & 245 & - & 92.1 & -- & 109 & -- & 6 & -- & 33 \\
\hline 290 & 8 & 10 & 244 & -- & 103 & -- & 117 & - & 6.2 & -- & 35.6 \\
\hline 267 & 130 & 660 & 203 & - & 98.9 & -- & 154 & -- & 7.5 & -- & 29 \\
\hline 290 & 25 & $<10$ & 243 & -- & 98.2 & -- & 116 & -- & 7.7 & -- & 32.9 \\
\hline- & 28 & 1,100 & 235 & -- & 94.7 & -- & 99.7 & -- & 6.7 & - & 32.7 \\
\hline- & 256 & 1,200 & 180 & -- & 87.8 & -- & 123 & -- & 9.7 & -- & 22.4 \\
\hline 248 & 274 & 900 & 138 & -- & 51.3 & -- & 64.6 & -- & 6.9 & -- & 15 \\
\hline 260 & 34 & 460 & 252 & - & 91.7 & -- & 86.1 & -- & 7.4 & - & 30.9 \\
\hline 264 & 31 & 260 & 261 & - & 104 & - & 99.5 & -- & 7.4 & -- & 37.2 \\
\hline 275 & 21 & 70 & 250 & -- & 105 & -- & 108 & - & 7 & -- & 39 \\
\hline 289 & 9 & 30 & 261 & -- & 109 & -- & 117 & -- & 6.5 & -- & 40.1 \\
\hline 314 & 9 & 280 & 259 & - & 108 & -- & 110 & -- & 7.1 & -- & 39.5 \\
\hline 296 & 14 & 270 & 260 & -- & 99.5 & -- & 107 & -- & 6.6 & -- & 39.4 \\
\hline 360 & 12 & 36 & 283 & - & 111 & -- & 114 & -- & 6.6 & -- & 40.9 \\
\hline 275 & 16 & $<2$ & 251 & -- & 97.2 & -- & 106 & -- & 5.7 & -- & 38.3 \\
\hline 283 & 41 & $<10$ & 237 & -- & 98.8 & -- & 121 & -- & 7.2 & -- & 33.6 \\
\hline 277 & 40 & 10 & 234 & - & 98.3 & -- & 120 & -- & 7.1 & -- & 33.5 \\
\hline-- & 39 & -- & 232.45 & -- & 97.95 & -- & 124.4 & -- & 7.23 & -- & 36.62 \\
\hline 295 & 23 & 30 & 248 & - & 109 & -- & 120 & -- & 6.9 & -- & 38.7 \\
\hline 288 & 102 & 470 & 227 & - & 94.1 & -. & 121 & -- & 7.7 & -- & 33 \\
\hline 288 & 63 & 380 & 223 & - & 94.1 & -- & 121 & -- & 7.1 & -- & 33.5 \\
\hline
\end{tabular}


Table 6. Water-quality data for selected sites along Bear Butte Creek-Continued

$\left[\mathrm{ft}^{3} / \mathrm{s}\right.$, cubic feet per second; $\mu \mathrm{S} / \mathrm{cm}$, microsiemens per centimeter; deg C, degrees Celsius; $\mathrm{mg} / \mathrm{L}$, milligrams per liter; $\mu \mathrm{g} / \mathrm{L}$, micrograms per liter: cols, colonies; $\mathrm{mL}$, milliliters; $\mathrm{K}$. non-ideal colony count: <. less than; --. no data available]

\begin{tabular}{|c|c|c|c|c|c|c|c|c|c|c|c|}
\hline Date & Time & $\begin{array}{l}\text { Dis- } \\
\text { solved } \\
\text { sulfate } \\
\text { (mg/L) }\end{array}$ & $\begin{array}{c}\text { Total } \\
\text { sulfate } \\
\text { (mg/L) }\end{array}$ & $\begin{array}{l}\text { Dis- } \\
\text { solved } \\
\text { fluoride } \\
\text { (mg/L) }\end{array}$ & $\begin{array}{c}\text { Total } \\
\text { fluoride } \\
\text { (mg/L) }\end{array}$ & $\begin{array}{c}\text { Nitrate } \\
\text { as nitro- } \\
\text { gen } \\
(\mathrm{mg} / \mathrm{L})\end{array}$ & $\begin{array}{c}\text { Nitrogen } \\
\text { ammonia } \\
(\mathrm{mg} / \mathrm{L})\end{array}$ & $\begin{array}{c}\text { Total } \\
\text { kjeldahl } \\
\text { nitrogen } \\
(\mathrm{mg} / \mathrm{L})\end{array}$ & $\begin{array}{l}\text { Total } \\
\text { dissolved } \\
\text { phos- } \\
\text { phorus } \\
\text { (mg/L) }\end{array}$ & $\begin{array}{l}\text { Dis- } \\
\text { solved } \\
\text { cyanide } \\
\text { (mg/L) }\end{array}$ & $\begin{array}{c}\text { Dis- } \\
\text { sovled } \\
\text { arsenic } \\
(\mu \mathrm{g} / \mathrm{L})\end{array}$ \\
\hline \multicolumn{12}{|c|}{ Site 3 - 06437500 Bear Butte Creek near Sturgis, SD—Continued } \\
\hline $06-16-1998$ & 1315 & -- & 938 & - & 0.39 & 0.4 & $<0.02$ & 0.41 & 0.125 & $<0.01$ & 3 \\
\hline 06-18-1998 & 1532 & -- & 692 & -- & .42 & .4 & $<.02$ & 2.05 & 1.64 & $<.01$ & 1.6 \\
\hline $07-07-1998$ & 1240 & -- & 948 & - & .41 & .3 & $<.02$ & 1.19 & .144 & $<.01$ & 2.3 \\
\hline $08-11-1998$ & 1340 & -- & 1,023 & -- & .39 & .3 & $<.02$ & .86 & .066 & $<.01$ & 3.4 \\
\hline 09-16-1998 & 1230 & -- & 1,059 & -- & .38 & .2 & $<.02$ & .66 & .039 & $<.01$ & 1.5 \\
\hline $10-07-1998$ & 1035 & -- & 450 & -- & .32 & .3 & $<.02$ & 1.38 & .358 & $<.01$ & 1.7 \\
\hline${ }^{3} 10-07-1998$ & 1036 & -- & 449 & - & .32 & .4 & $<.02$ & 1.09 & .353 & $<.01$ & 2.1 \\
\hline $11-17-1998$ & 1250 & -- & 752 & - & .42 & .5 & $<.02$ & .67 & .058 & $<.01$ & 1.3 \\
\hline $12-15-1998$ & 1250 & 923 & - & - & -- & 1.1 & $<.02$ & .56 & .024 & $<.01$ & 2.7 \\
\hline 01-26-1999 & 0855 & 964 & -- & 0.41 & -- & 1.3 & $<.02$ & .34 & .018 & $<.01$ & 1.6 \\
\hline 02-10-1999 & 1230 & 947 & - & .38 & - & 1 & $<.02$ & .46 & .024 & $<.01$ & 1.4 \\
\hline 03-09-1999 & 1245 & 1,022 & - & .37 & -- & .8 & $<.02$ & .48 & .026 & $<.01$ & 1.2 \\
\hline 04-06-1999 & 1220 & 955 & - & .39 & - & .2 & $<.02$ & 1.05 & .174 & $<.01$ & 2.3 \\
\hline 04-21-1999 & 1235 & 946 & -- & .4 & -- & .4 & $<.02$ & .67 & .049 & $<.01$ & 2 \\
\hline 05-10-1999 & 1310 & 898 & -- & .38 & -- & .7 & $<.02$ & .55 & .014 & $<.01$ & .7 \\
\hline 06-09-1999 & 1245 & 838 & - & - & - & .3 & $<.02$ & 1.1 & .424 & $<.01$ & 2.4 \\
\hline 06-15-1999 & 1105 & 431 & -- & - & -- & .5 & $<.02$ & 1.31 & .372 & $<.01$ & 2.9 \\
\hline 07-19-1999 & 1050 & 898 & -- & - & -- & .9 & $<.02$ & .75 & .062 & $<.01$ & 2.4 \\
\hline 08-16-1999 & 1145 & 974 & -- & -- & - & .8 & $<.02$ & .65 & .048 & $<.01$ & 2 \\
\hline 09-14-1999 & 1245 & 1,059 & -- & .26 & -- & 1 & $<.02$ & .51 & .026 & $<.01$ & 1.4 \\
\hline $10-26-1999$ & 1220 & 1,057 & -- & .34 & -- & .7 & $<.02$ & .36 & .02 & $<.01$ & $<1$ \\
\hline $11-16-1999$ & 1155 & 1,036 & -- & .26 & -- & .8 & $<.02$ & .45 & .014 & $<.01$ & $<1$ \\
\hline 12-07-1999 & 1205 & 963 & -- & .34 & -- & 1.1 & $<.02$ & .39 & .018 & $<.01$ & 1.3 \\
\hline $01-18-2000$ & 1255 & 1,081 & -- & .41 & -- & 1.3 & $<.02$ & .44 & .016 & $<.01$ & $<1$ \\
\hline $02-22-2000$ & 1200 & 991 & - & .37 & - & 1.1 & $<.02$ & .44 & .024 & $<.01$ & 1.3 \\
\hline $03-28-2000$ & 1400 & 974 & -- & .42 & -- & .3 & $<.02$ & .58 & .074 & $<.01$ & 1.2 \\
\hline${ }^{3} 03-28-2000$ & 1402 & 970 & -- & .43 & -- & .3 & $<.02$ & .5 & .07 & $<.01$ & 1.2 \\
\hline${ }^{2} 03-28-2000$ & 1405 & $1,016.1$ & -- & .346 & -- & .307 & $<.2$ & .414 & .058 & $<.01$ & 1.29 \\
\hline $04-04-2000$ & 1200 & 1,000 & -- & .39 & -- & .5 & $<.02$ & .58 & .041 & $<.01$ & 1 \\
\hline $05-09-2000$ & 1215 & 905 & -- & .41 & - & .4 & $<.02$ & .74 & .133 & $<.01$ & 1.7 \\
\hline $05-18-2000$ & 1230 & 906 & -- & .4 & -- & .5 & $<.02$ & .93 & .104 & $<.01$ & 1.4 \\
\hline
\end{tabular}




\begin{tabular}{|c|c|c|c|c|c|c|c|c|c|c|c|}
\hline $\begin{array}{c}\text { Dis- } \\
\text { solved } \\
\text { cadmium } \\
(\mu \mathrm{g} / \mathrm{L})\end{array}$ & $\begin{array}{l}\text { Dis- } \\
\text { solved } \\
\text { chro- } \\
\text { mium } \\
(\mu \mathrm{g} / \mathrm{L})\end{array}$ & $\begin{array}{l}\text { Dis- } \\
\text { solved } \\
\text { copper } \\
(\mu \mathrm{g} / \mathrm{L})\end{array}$ & $\begin{array}{l}\text { Dis- } \\
\text { solved } \\
\text { iron } \\
(\mathrm{mg} / \mathrm{L})\end{array}$ & $\begin{array}{c}\text { Total } \\
\text { iron } \\
\text { (mg/L) }\end{array}$ & $\begin{array}{c}\text { Dis- } \\
\text { solved } \\
\text { lead } \\
(\mu \mathrm{g} / \mathrm{L})\end{array}$ & $\begin{array}{c}\text { Dis- } \\
\text { solved } \\
\text { manga- } \\
\text { nese } \\
(\mathrm{mg} / \mathrm{L})\end{array}$ & $\begin{array}{c}\text { Total } \\
\text { manga- } \\
\text { neses } \\
(\mathrm{mg} / \mathrm{L})\end{array}$ & $\begin{array}{c}\text { Dis- } \\
\text { solved } \\
\text { mercury } \\
(\mu \mathrm{g} / \mathrm{L})\end{array}$ & $\begin{array}{l}\text { Dis- } \\
\text { solved } \\
\text { nickel } \\
(\mu \mathrm{g} / \mathrm{L})\end{array}$ & $\begin{array}{l}\text { Dis- } \\
\text { solved } \\
\text { silver } \\
(\mu \mathrm{g} / \mathrm{L})\end{array}$ & $\begin{array}{c}\text { Dis- } \\
\text { solved } \\
\text { zinc } \\
(\mu \mathrm{g} / \mathrm{L})\end{array}$ \\
\hline$<0.5$ & 8.7 & $<0.02$ & -- & 2.22 & 1.5 & -- & 0.19 & $<0.2$ & 7.4 & $<0.5$ & $<50$ \\
\hline$<.2$ & 37.9 & $<.02$ & -- & 39 & $<.8$ & -- & 1.6 & $<.2$ & 2.4 & $<.5$ & $<50$ \\
\hline$<.1$ & 7.8 & $<.02$ & -- & 1.92 & $<.8$ & -- & .2 & $<.13$ & 7.9 & $<.2$ & $<50$ \\
\hline$<.1$ & 3 & $<.02$ & -- & .45 & $<.8$ & -- & .05 & $<.2$ & 8.4 & $<.2$ & $<3$ \\
\hline$<.1$ & 7.2 & $<.02$ & -- & .2 & $<1$ & -- & .02 & $<.2$ & 7.6 & $<.2$ & $<3$ \\
\hline$<.1$ & $<1$ & $<.02$ & -- & 5.2 & $<1$ & -- & .24 & $<.2$ & 5 & $<1$ & $<3$ \\
\hline$<.1$ & $<1$ & $<.02$ & -- & 5.26 & $<1$ & -- & .24 & $<.2$ & 4.8 & $<1$ & $<3$ \\
\hline$<.2$ & 4 & $<.02$ & -- & $<.06$ & $<.8$ & -- & .08 & $<.2$ & 8.6 & $<.2$ & 5.1 \\
\hline$<.2$ & 3.7 & $<.02$ & -- & -- & $<.1$ & -- & -- & $<.1$ & 9.9 & $<.2$ & 4 \\
\hline$<.2$ & 5.4 & $<.02$ & 0.04 & -- & $<.8$ & 0.08 & - & $<.1$ & 9 & $<.2$ & $<40$ \\
\hline$<.2$ & 3.5 & 1.8 & .02 & -- & $<.1$ & .09 & -- & $<.1$ & 10.5 & $<.2$ & 6 \\
\hline$<.2$ & 6 & 1.5 & - & -- & $<.1$ & -- & - & $<.1$ & 7.5 & $<.2$ & 4 \\
\hline$<.2$ & 7.1 & 2 & -- & -- & $<.1$ & -- & -- & $<.1$ & 7.6 & $<.2$ & $<3$ \\
\hline$<.2$ & 5.7 & 1.8 & - & -- & $<.1$ & -- & -- & $<.1$ & 9.3 & $<.2$ & 6 \\
\hline$<.2$ & 8.2 & 1.5 & .03 & -- & $<.1$ & .09 & -- & $<.1$ & 9 & $<.2$ & 5 \\
\hline$<.2$ & $<1.6$ & $<.02$ & $<.02$ & -- & $<.1$ & .05 & - & $<.1$ & 9 & $<.2$ & 4 \\
\hline$<.2$ & 4.4 & 1.7 & -- & -- & $<.1$ & -- & -- & $<.1$ & 6.8 & $<.2$ & $<3$ \\
\hline$<.2$ & 7 & 1.6 & -- & -- & .1 & -- & -- & .1 & 7.8 & 2.4 & 6 \\
\hline$<.2$ & 6.7 & 2.6 & $<.02$ & -- & $<.1$ & .05 & - & $<.1$ & 7.9 & $<.2$ & 6 \\
\hline$<.2$ & 4.8 & 1.3 & .04 & -- & $<.1$ & .03 & -- & $<.1$ & 9.3 & $<.2$ & 6 \\
\hline$<.2$ & 4.8 & 1.4 & $<.02$ & -- & $<.1$ & $<.02$ & -- & .1 & 8.2 & $<.2$ & 5 \\
\hline$<.2$ & 3.6 & 1.1 & .03 & - & $<.1$ & .05 & -- & $<.1$ & 8.2 & $<.2$ & 5 \\
\hline$<.2$ & 8 & 1.1 & $<.02$ & -- & $<.1$ & .05 & -- & $<.1$ & 8.2 & $<.2$ & 6 \\
\hline$<.2$ & 5.9 & 1.5 & .04 & -- & $<.1$ & .06 & -- & $<.1$ & 8.4 & $<.2$ & 4 \\
\hline$<.2$ & 7.6 & 1.4 & $<.02$ & -- & $<.1$ & .05 & -- & $<.1$ & 9.6 & $<.2$ & 4 \\
\hline$<.2$ & 5 & 2 & .03 & -- & $<.1$ & .1 & -- & $<.1$ & 9.9 & $<.2$ & $<3$ \\
\hline$<.2$ & 6.2 & 1.9 & .03 & -- & $<.1$ & .1 & -- & $<.1$ & 9.2 & $<.2$ & $<3$ \\
\hline$<1$ & $<1$ & 2.08 & $<.03$ & -. & $<1$ & .104 & -- & $<.23$ & 1.23 & $<1$ & 39 \\
\hline$<.2$ & 7.2 & 2 & .02 & -- & $<.1$ & .0926 & -- & $<.1$ & 9.5 & $<.2$ & $<3$ \\
\hline$<.2$ & 2.9 & 1.7 & .02 & - & $<.1$ & .07 & -- & $<.1$ & 8.9 & $<.2$ & 3 \\
\hline$<.2$ & 12.6 & 1.6 & .04 & -- & $<.1$ & .06 & -- & $<.1$ & 8.8 & $<.2$ & 3 \\
\hline
\end{tabular}


Table 6. Water-quality data for selected sites along Bear Butte Creek-Continued

$\left[\mathrm{ft}^{3} / \mathrm{s}\right.$, cubic feet per second; $\mu \mathrm{S} / \mathrm{cm}$, microsiemens per centimeter; deg C, degrees Celsius; $\mathrm{mg} / \mathrm{L}$, milligrams per liter; $\mu \mathrm{g} / \mathrm{L}$, micrograms per liter; cols, colonies; $\mathrm{mL}$, milliliters; $\mathrm{K}$, non-ideal colony count; <, less than; --, no data available]

\begin{tabular}{|c|c|c|c|c|c|c|c|c|c|c|c|}
\hline Date & Time & $\begin{array}{c}\text { Dis- } \\
\text { charge } \\
\left(\mathrm{ft}^{3} / \mathrm{s}\right)\end{array}$ & $\begin{array}{c}\text { Specific } \\
\text { conduc- } \\
\text { tance } \\
(\mu \mathrm{S} / \mathrm{cm})\end{array}$ & $\begin{array}{c}\mathrm{pH} \\
\text { (standard } \\
\text { units) }\end{array}$ & $\begin{array}{c}\text { Water } \\
\text { tempera- } \\
\text { ture } \\
\text { (deg C) }\end{array}$ & $\begin{array}{c}\text { Air } \\
\text { tempera- } \\
\text { ture } \\
(\operatorname{deg} \mathrm{C})\end{array}$ & $\begin{array}{c}\text { Dis- } \\
\text { solved } \\
\text { oxygen } \\
\text { (mg/L) }\end{array}$ & $\begin{array}{c}\text { Bio- } \\
\text { logical } \\
\text { oxygen } \\
\text { demand } \\
\text { (mg/L) }\end{array}$ & $\begin{array}{c}\text { Carbon } \\
\text { oxygen } \\
\text { demand } \\
\text { (mg/L) }\end{array}$ & $\begin{array}{c}\text { Hardness } \\
\text { as } \mathrm{CaCO}_{3} \\
(\mathrm{mg} / \mathrm{L})\end{array}$ & $\begin{array}{l}\text { Labora- } \\
\text { tory } \\
\text { alkalinity } \\
\text { (mg/L) }\end{array}$ \\
\hline \multicolumn{12}{|c|}{ Site 4 - 443048103091400 Bear Butte Creek near mouth, near Vale, SD } \\
\hline 06-16-1998 & 1455 & 7.3 & 2,170 & 7.9 & 20.0 & 25.5 & 10.9 & $<1$ & 13.2 & 986 & 195 \\
\hline $06-18-1998$ & 1745 & 529 & 1,610 & 7.7 & 13.0 & 16.5 & 7.4 & 14 & 22.7 & 881 & 317 \\
\hline 07-07-1998 & 1415 & 12 & 2,140 & 8.1 & 27.5 & 31.0 & 9.1 & 3 & 19 & 937 & 195 \\
\hline 08-11-1998 & 1450 & 3.1 & 2,140 & 8.1 & 32.0 & 27.5 & 8.6 & $<2$ & 18.7 & 934 & 181 \\
\hline 09-16-1998 & 1245 & -- & -- & -- & -- & -- & -- & -- & -- & -- & -- \\
\hline $10-07-1998$ & 1330 & 56 & 1,980 & 7.6 & 8.0 & 19.5 & 12.3 & $<2$ & 21.7 & 970 & 193 \\
\hline${ }^{3} 10-07-1998$ & 1331 & 56 & 1,980 & 7.6 & 8.0 & 19.5 & 12.3 & $<2$ & 19.8 & 968 & 192 \\
\hline $11-17-1998$ & 1420 & 62.0 & 1,890 & 8.0 & 4.0 & 3.5 & 13.6 & $<1$ & $<20$ & 809 & 207 \\
\hline $12-15-1998$ & 1430 & 22.0 & 2,080 & 7.8 & .0 & 5.5 & 16.5 & $<1$ & $<20$ & 990 & 244 \\
\hline 01-26-1999 & 1125 & 14 & 2,360 & 8.0 & .0 & -2.0 & 13.5 & $<1$ & 13.6 & 1,122 & 279 \\
\hline 02-10-1999 & 1405 & 21 & 2,220 & 8.1 & .0 & 2.5 & 12.4 & $<1$ & 21.1 & 942 & 216 \\
\hline 03-09-1999 & 1435 & 20 & 2,310 & 8.0 & 3.0 & 3.5 & 14.2 & $<1$ & 11.6 & 1,000 & 218 \\
\hline 04-06-1999 & 1400 & 107 & 2,150 & 8.1 & 8.5 & 16.0 & 9.6 & 3 & 23.3 & 786 & 212 \\
\hline 04-21-1999 & 1333 & 42 & 2,620 & 8.1 & 11.0 & 7.0 & 10.8 & $<1$ & 22 & 1,024 & 248 \\
\hline 05-10-1999 & 1445 & 37 & 2,410 & 8.1 & 9.0 & 5.0 & 5.3 & $<1$ & 22.2 & 1,004 & 244 \\
\hline 06-09-1999 & 1430 & 119 & 1,930 & 8.2 & 21.0 & 22.0 & 8.2 & 3 & 26.7 & 754 & 215 \\
\hline 06-15-1999 & 1350 & 265 & 1,410 & 8.3 & 18.0 & 21.0 & 5.1 & $<2$ & 19.4 & 570 & 211 \\
\hline 07-19-1999 & 1210 & 32 & 2,070 & 7.9 & 23.5 & 30.5 & 6.1 & $<1$ & 14.7 & 955 & 204 \\
\hline 08-16-1999 & 1255 & 12 & 2,090 & 8.0 & 23.0 & 26.0 & 8.9 & $<1$ & 13.7 & 1,040 & 192 \\
\hline 09-14-1999 & 1430 & 7.9 & 2,250 & 7.7 & 15.5 & 18.5 & 9.0 & $<1$ & 12.7 & 1,052 & 199 \\
\hline $10-26-1999$ & 1345 & 12 & 2,350 & 8.1 & 9.0 & 23.0 & 11.6 & $<1$ & $<10$ & 1,079 & 214 \\
\hline $11-16-1999$ & 1325 & 11 & 2,300 & 8.1 & 5.5 & 12.0 & 11.9 & $<1$ & 13.2 & 1,090 & 235 \\
\hline $12-07-1999$ & 1340 & 16 & 2,400 & 7.7 & .5 & 6.0 & 13.2 & $<1$ & $<10$ & 1,092 & 236 \\
\hline $01-18-2000$ & 1425 & 11 & 2,540 & 8.1 & .0 & 7.5 & 14.2 & $<1$ & 13.5 & 1,166 & 281 \\
\hline $02-22-2000$ & 1330 & 13 & 2,280 & 8.2 & .5 & 20.0 & 14.5 & $<1$ & $<10$ & 971 & 211 \\
\hline $03-29-2000$ & 1000 & 20 & 2,240 & 8.2 & 7.5 & 7.0 & 11.2 & $<1$ & 11.6 & 1.010 & 221 \\
\hline 04-04-2000 & 1330 & 13 & 2,300 & 8.1 & 8.0 & 20.0 & 11.4 & $<1$ & $<10$ & 1,060 & 220 \\
\hline 05-09-2000 & 1345 & 53 & 2,120 & 8.4 & 14.5 & 18.5 & 10.4 & $<2$ & 21.2 & 879 & 234 \\
\hline $05-18-2000$ & 1400 & 37 & 2,220 & 8.5 & 14.5 & 14.0 & 10.6 & $<2$ & 23.5 & 950 & 237 \\
\hline
\end{tabular}




\begin{tabular}{|c|c|c|c|c|c|c|c|c|c|c|c|}
\hline $\begin{array}{c}\text { Bicar- } \\
\text { bonate as } \\
\mathrm{CaCO}_{3} \\
(\mathrm{mg} / \mathrm{L})\end{array}$ & $\begin{array}{l}\text { Total } \\
\text { sus- } \\
\text { pended } \\
\text { solids } \\
\text { (mg/L) }\end{array}$ & $\begin{array}{c}\text { Fecal } \\
\text { coliform } \\
\text { (cols/ } \\
100 \mathrm{~mL} \text { ) }\end{array}$ & $\begin{array}{c}\text { Dis- } \\
\text { solved } \\
\text { calcium } \\
(\mathrm{mg} / \mathrm{L})\end{array}$ & $\begin{array}{l}\text { Total } \\
\text { calcium } \\
\text { (mg/L) }\end{array}$ & $\begin{array}{c}\text { Dis- } \\
\text { solved } \\
\text { magne- } \\
\text { sium } \\
(\mathrm{mg} / \mathrm{L})\end{array}$ & $\begin{array}{c}\text { Total } \\
\text { magne- } \\
\text { sium } \\
(\mathrm{mg} / \mathrm{L})\end{array}$ & $\begin{array}{c}\text { Dis- } \\
\text { solved } \\
\text { sodium } \\
\text { (mg/L) }\end{array}$ & $\begin{array}{l}\text { Total } \\
\text { sodium } \\
\text { (mg/L) }\end{array}$ & $\begin{array}{c}\text { Dis- } \\
\text { solved } \\
\text { potas- } \\
\text { sium } \\
\text { (mg/L) }\end{array}$ & $\begin{array}{c}\text { Total } \\
\text { potas- } \\
\text { sium } \\
(\mathrm{mg} / \mathrm{L})\end{array}$ & $\begin{array}{c}\text { Dis- } \\
\text { solved } \\
\text { chloride } \\
\text { (mg/L) }\end{array}$ \\
\hline & & & & & & & & & & & \\
\hline- & 46 & 20 & -- & 229 & -- & 100.7 & - & 131 & -- & 8.3 & 44 \\
\hline-- & 6,440 & 15.000 & -- & 218 & - & 82 & -- & 200 & -- & 40.2 & 7 \\
\hline-- & 29 & 80 & -- & 219 & -- & 94.8 & - & 136 & -- & 8.8 & 36 \\
\hline-- & 23 & 100 & -- & 213 & -- & 97.7 & - & 136 & -- & 8.3 & 44 \\
\hline-- & -- & -- & -- & -- & -- & -- & -- & -- & -- & -- & - \\
\hline- & 384 & 2,000 & -- & 247 & -- & 86 & -- & 100 & - & 10.8 & 23 \\
\hline- & 392 & 2,000 & - & 245 & -- & 86.5 & -- & 102 & -- & 11.1 & 23 \\
\hline 253 & 40 & 110 & -- & 187 & -- & 83.2 & -- & 139 & -- & 8.6 & 33 \\
\hline 298 & 14 & $<10$ & 240 & - & 94.9 & -- & 133 & -- & 6.9 & -- & 42 \\
\hline 340 & 12 & $<10$ & 278 & -- & 104.2 & -- & 131 & -- & 7.2 & -- & 39.6 \\
\hline 263 & 30 & 40 & 224 & -- & 93.1 & - & 149 & -- & 6.5 & -- & 39.7 \\
\hline 266 & 15 & $<10$ & 230 & -- & 105 & -- & 157 & - & 6.3 & - & 41.8 \\
\hline 258 & 416 & 780 & 167 & -- & 89.7 & - & 195 & -- & 8.4 & -- & 43.9 \\
\hline 303 & 54 & $<10$ & 229 & -- & 110 & - & 218 & -- & 8.5 & - & 44.4 \\
\hline- & 52 & 220 & 224 & - & 108 & -- & 189 & -- & 7.6 & -- & 40.9 \\
\hline-- & 440 & 400 & 156 & -- & 88.6 & - & 157 & -- & 10.1 & -- & 31.5 \\
\hline 257 & 552 & 1,100 & 133 & -- & 57.9 & - & 95.9 & -- & 7.9 & - & 23.1 \\
\hline 249 & 42 & 150 & 224 & -- & 96.1 & - & 119 & -- & 7.9 & -. & 35.8 \\
\hline 234 & 36 & 40 & 241 & -- & 106 & -- & 127 & -- & 8.3 & -- & 40.2 \\
\hline-- & 24 & 60 & 235 & - & 113 & - & 136 & -- & 6.9 & -- & 40.7 \\
\hline 261 & 6 & $<10$ & 246 & -- & 113 & -- & 148 & -- & 7.7 & -. & 43.5 \\
\hline 287 & 4 & $<10$ & 247 & -- & 115 & - & 143 & -- & 7.6 & -- & 43.8 \\
\hline 288 & 14 & 26 & 251 & -- & 113 & -- & 148 & -- & 7.7 & -- & 43.8 \\
\hline 343 & 6 & 12 & 274 & -- & 117 & -- & 151 & -- & 7.1 & -- & 45.8 \\
\hline 257 & 18 & $<2$ & 228 & - & 97.7 & - & 136 & -- & 5.9 & -- & 41.1 \\
\hline 270 & 31 & $<10$ & 229 & -- & 107 & - & 149 & -- & 7.6 & & 36.6 \\
\hline 268 & 12 & $<10$ & 243 & -- & 110 & - & 152 & -- & 7.3 & -- & 41.8 \\
\hline 285 & 118 & 600 & 196 & -- & 94.7 & - & 174 & -- & 8.7 & -- & 40.9 \\
\hline 287 & 70 & 160 & 214 & -- & 101 & -- & 175 & -- & 8 & -- & 39.5 \\
\hline
\end{tabular}


Table 6. Water-quality data for selected sites along Bear Butte Creek-Continued

$\left[\mathrm{ft}^{3} / \mathrm{s}\right.$, cubic feet per second; $\mu \mathrm{S} / \mathrm{cm}$, microsiemens per centimeter; deg $\mathrm{C}$, degrees Celsius; $\mathrm{mg} / \mathrm{L}$, milligrams per liter; $\mu \mathrm{g} / \mathrm{L}$, micrograms per liter; cols, colonies; mL. milliliters: $\mathrm{K}$, non-ideal colony count; <, less than; --, no data available]

\begin{tabular}{|c|c|c|c|c|c|c|c|c|c|c|c|}
\hline Date & Time & $\begin{array}{l}\text { Dis- } \\
\text { solved } \\
\text { sulfate } \\
\text { (mg/L) }\end{array}$ & $\begin{array}{c}\text { Total } \\
\text { sulfate } \\
\text { (mg/L) }\end{array}$ & $\begin{array}{c}\text { Dis- } \\
\text { solved } \\
\text { fluoride } \\
\text { (mg/L) }\end{array}$ & $\begin{array}{c}\text { Total } \\
\text { fluoride } \\
\text { (mg/L) }\end{array}$ & $\begin{array}{c}\text { Nitrate } \\
\text { as nitro- } \\
\text { gen } \\
(\mathrm{mg} / \mathrm{L})\end{array}$ & $\begin{array}{c}\text { Nitrogen } \\
\text { ammonia } \\
(\mathrm{mg} / \mathrm{L})\end{array}$ & $\begin{array}{c}\text { Total } \\
\text { kjeldahl } \\
\text { nitrogen } \\
\text { (mg/L) }\end{array}$ & $\begin{array}{c}\text { Total } \\
\text { dissolved } \\
\text { phos- } \\
\text { phorus } \\
\text { (mg/L) }\end{array}$ & $\begin{array}{c}\text { Dis- } \\
\text { solved } \\
\text { cyanide } \\
\text { (mg/L) }\end{array}$ & $\begin{array}{c}\text { Dis- } \\
\text { sovled } \\
\text { arsenic } \\
\text { ( } \mu \mathrm{g} / \mathrm{L})\end{array}$ \\
\hline \multicolumn{12}{|c|}{ Site 4 - 443048103091400 Bear Butte Creek near mouth, near Vale, SD-Continued } \\
\hline 06-16-1998 & 1455 & -- & 1,052 & -- & 0.37 & $<0.1$ & $<0.02$ & 0.64 & 0.072 & $<0.01$ & 3 \\
\hline $06-18-1998$ & 1745 & -- & 717 & - & .43 & .5 & .19 & 2.26 & 4.04 & $<.01$ & 2 \\
\hline 07-07-1998 & 1415 & -- & 992 & -- & .38 & $<.1$ & $<.02$ & 1.08 & .072 & $<.01$ & 1.7 \\
\hline 08-11-1998 & 1450 & - & 1,058 & -- & .37 & $<.1$ & $<.02$ & .83 & .051 & $<.01$ & 2.4 \\
\hline 09-16-1998 & 1245 & -- & -- & -- & -- & -- & -- & -- & -- & -- & -- \\
\hline $10-07-1998$ & 1330 & - & 996 & -- & .42 & .3 & $<.02$ & 1.35 & .554 & $<.01$ & 2 \\
\hline${ }^{3} 10-07-1998$ & 1331 & -- & 992 & -- & .42 & .3 & $<.02$ & 1.4 & .559 & $<.01$ & 1.8 \\
\hline $11-17-1998$ & 1420 & -- & 836 & -- & .42 & .5 & $<.02$ & .73 & .086 & $<.01$ & 1.2 \\
\hline $12-15-1998$ & 1430 & 931 & -- & 0.37 & -- & 1 & $<.02$ & .53 & .021 & $<.01$ & 2.3 \\
\hline 01-26-1999 & 1125 & 1,082 & - & .43 & -- & 1.2 & $<.02$ & .48 & .025 & $<.01$ & 1.7 \\
\hline 02-10-1999 & 1405 & 969 & - & .37 & -- & .8 & $<.02$ & .5 & .057 & $<.01$ & 1.3 \\
\hline 03-09-1999 & 1435 & 1,044 & -- & .39 & -- & .6 & $<.02$ & .56 & .029 & $<.01$ & 1.5 \\
\hline 04-06-1999 & 1400 & 679 & -- & .33 & -- & .3 & $<.02$ & .96 & .317 & $<.01$ & 2.6 \\
\hline 04-21-1999 & 1333 & 1,175 & -- & .41 & -- & .4 & $<.02$ & .66 & .069 & $<.01$ & 1.9 \\
\hline 05-10-1999 & 1445 & 1,123 & -- & .4 & -- & .6 & $<.02$ & .59 & .051 & $<.01$ & 1 \\
\hline 06-09-1999 & 1430 & 844 & -- & -- & -- & .2 & $<.02$ & 1.17 & .576 & $<.01$ & 2.2 \\
\hline 06-15-1999 & 1350 & 529 & - & - & -- & .6 & $<.02$ & 1.25 & .676 & $<.01$ & 2.2 \\
\hline 07-19-1999 & 1210 & 954 & -- & -- & -- & .7 & $<.02$ & .4 & .059 & $<.01$ & 2.1 \\
\hline 08-16-1999 & 1255 & 1,001 & -- & - & -- & .5 & $<.02$ & .54 & .052 & $<.01$ & 1.5 \\
\hline 09-14-1999 & 1430 & 1,103 & -- & .27 & -- & .6 & $<.02$ & .41 & .028 & $<.01$ & 1.4 \\
\hline $10-26-1999$ & 1345 & 1,145 & -- & .33 & -- & .5 & $<.02$ & .38 & .011 & $<.01$ & $<1$ \\
\hline $11-16-1999$ & 1325 & 1,126 & -- & .36 & -- & .6 & $<.02$ & .39 & .009 & $<.01$ & 1 \\
\hline 12-07-1999 & 1340 & 1,111 & -- & .34 & -- & .8 & $<.02$ & .41 & .02 & $<.01$ & 1.2 \\
\hline $01-18-2000$ & 1425 & 1,165 & -- & .39 & -- & 1.1 & $<.02$ & .45 & .005 & $<.01$ & $<1$ \\
\hline $02-22-2000$ & 1330 & 1,020 & -- & .36 & -- & .9 & $<.02$ & .33 & .022 & $<.01$ & 1.1 \\
\hline $03-29-2000$ & 1000 & 1,045 & -- & .39 & -- & .1 & $<.02$ & .66 & .042 & $<.01$ & 1.2 \\
\hline $04-04-2000$ & 1330 & 1,110 & -- & .38 & -- & .2 & $<.02$ & .52 & .022 & $<.01$ & 1 \\
\hline $05-09-2000$ & 1345 & 923 & -- & .38 & -- & .3 & $<.02$ & .94 & .187 & $<.01$ & 1.9 \\
\hline $05-18-2000$ & 1400 & 1,020 & -- & .41 & -- & .4 & $<.02$ & .9 & .109 & $<.01$ & 1.5 \\
\hline
\end{tabular}

${ }^{\mathrm{I}}$ Quality-assurance/quality-control blank sample analyzed at South Dakota State Health Laboratory.

${ }^{2}$ Quality-assurance/quality-control split sample analyzed at U.S. Geological Survey National Water Quality Laboratory.

${ }^{3}$ Quality-assurance/quality-control split sample analyzed at South Dakota State Health Laboratory. 


\begin{tabular}{|c|c|c|c|c|c|c|c|c|c|c|c|}
\hline $\begin{array}{c}\text { Dis- } \\
\text { solved } \\
\text { cadmium } \\
(\mu \mathrm{g} / \mathrm{L})\end{array}$ & $\begin{array}{l}\text { Dis- } \\
\text { solved } \\
\text { chro- } \\
\text { mium } \\
(\mu \mathrm{g} / \mathrm{L})\end{array}$ & $\begin{array}{c}\text { Dis- } \\
\text { solved } \\
\text { copper } \\
(\mu \mathrm{g} / \mathrm{L})\end{array}$ & $\begin{array}{l}\text { Dis- } \\
\text { solved } \\
\text { iron } \\
(\mathrm{mg} / \mathrm{L})\end{array}$ & $\begin{array}{c}\text { Total } \\
\text { iron } \\
(\mathrm{mg} / \mathrm{L})\end{array}$ & $\begin{array}{c}\text { Dis- } \\
\text { solved } \\
\text { lead } \\
\left(\mu g^{\prime} L\right)\end{array}$ & $\begin{array}{c}\text { Dis- } \\
\text { solved } \\
\text { manga- } \\
\text { nese } \\
(\mathrm{mg} / \mathrm{L})\end{array}$ & $\begin{array}{c}\text { Total } \\
\text { manga- } \\
\text { neses } \\
(\mathrm{mg} / \mathrm{L})\end{array}$ & $\begin{array}{c}\text { Dis- } \\
\text { solved } \\
\text { mercury } \\
(\mu \mathrm{g} / \mathrm{L})\end{array}$ & $\begin{array}{c}\text { Dis- } \\
\text { solved } \\
\text { nickel } \\
(\mu \mathrm{g} / \mathrm{L})\end{array}$ & $\begin{array}{c}\text { Dis- } \\
\text { solved } \\
\text { silver } \\
(\mu \mathrm{g} / \mathrm{L})\end{array}$ & $\begin{array}{c}\text { Dis- } \\
\text { solved } \\
\text { zinc } \\
(\mu \mathrm{g} / \mathrm{L})\end{array}$ \\
\hline$<0.5$ & 50.5 & $<0.02$ & -- & 0.96 & $<0.8$ & -- & 0.1 & 0.2 & 3.2 & $<0.5$ & $<50$ \\
\hline$<.5$ & 28.9 & $<.02$ & -- & 146 & $<.8$ & -- & 3.9 & $<.2$ & 4.4 & $<.5$ & $<50$ \\
\hline$<.1$ & 6.7 & $<.02$ & -- & .58 & $<.8$ & -- & .14 & $<.13$ & 7.9 & $<.2$ & $<50$ \\
\hline$<.1$ & 5.3 & $<.02$ & -- & .43 & $<.8$ & -- & .05 & $<.2$ & 7.6 & $<.2$ & $<3$ \\
\hline -- & -- & -- & -- & -- & .. & -- & -- & - & -- & -- & -- \\
\hline$<.1$ & $<.1$ & $<.02$ & -- & 10.1 & $<1$ & -- & .34 & $<.2$ & 8 & $<1$ & $<3$ \\
\hline$<.1$ & $<1$ & $<.02$ & -- & 9.8 & $<1$ & -- & .34 & $<.2$ & 7.7 & $<1$ & $<3$ \\
\hline$<.2$ & 2.6 & $<.02$ & -- & $<.06$ & $<.8$ & -- & .05 & $<.2$ & 7.4 & $<.2$ & 3.9 \\
\hline$<.2$ & 3.7 & $<.02$ & 0.17 & -- & $<.1$ & 0.03 & -- & $<.1$ & 9.7 & $<.2$ & 4 \\
\hline$<.2$ & 7 & $<.02$ & .02 & -- & $<.8$ & .08 & -- & $<.1$ & 10 & $<.2$ & $<40$ \\
\hline$<.2$ & 4.6 & 2.7 & .04 & -- & $<.1$ & .09 & - & $<.1$ & 10.2 & $<.2$ & 5 \\
\hline$<.2$ & 5.2 & 2.1 & -- & -- & $<.1$ & -- & -- & $<.1$ & 7.5 & $<.2$ & 12 \\
\hline$<.2$ & 7 & 3.1 & -- & -- & $<.1$ & -- & -- & .2 & 7.1 & .3 & $<3$ \\
\hline$<.2$ & 11.6 & 3.4 & -- & -- & $<.1$ & -- & -- & $<.1$ & 9.8 & $<.2$ & 4 \\
\hline$<.2$ & 7.5 & 2.7 & .02 & -- & $<.1$ & .06 & -- & $<.1$ & 9.1 & $<.2$ & 6 \\
\hline$<.2$ & 6.1 & $<.02$ & .02 & -- & $<.1$ & $<.02$ & -- & $<.1$ & 8.7 & $<.2$ & $<1$ \\
\hline$<.2$ & $<1.6$ & $<.02$ & $<.02$ & -- & $<.1$ & $<.02$ & - & .1 & 6.8 & .3 & $<3$ \\
\hline$<.2$ & 5.6 & 2 & -- & -- & $<.1$ & -- & -- & $<.1$ & 7.5 & $<.2$ & 4 \\
\hline$<.2$ & 6.6 & 2.1 & $<.02$ & -- & $<.1$ & .03 & - & $<.1$ & 7.6 & $<.2$ & 5 \\
\hline 1.2 & 4.2 & 1.9 & .02 & -- & .2 & .04 & -- & $<.1$ & 9.1 & $<.2$ & 10 \\
\hline$<.2$ & 4.3 & 1.9 & .02 & -- & $<.1$ & .04 & -- & .1 & 7.8 & $<.2$ & 4 \\
\hline$<.2$ & $<1$ & 1.4 & $<.02$ & -- & $<.1$ & .04 & - & $<.1$ & 8.2 & $<.2$ & 4 \\
\hline$<.2$ & 7.4 & 1.5 & .02 & - & $<.1$ & .05 & -- & $<.1$ & 8.3 & $<.2$ & 4 \\
\hline$<.2$ & 4.5 & 2.3 & .03 & -- & $<.1$ & .05 & -- & .1 & 10.5 & .3 & 4 \\
\hline$<.2$ & 5.6 & 1.9 & $<.02$ & -- & $<.1$ & .08 & - & $<.1$ & 9.5 & $<.2$ & 5 \\
\hline$<.2$ & 5 & 2.7 & $<.02$ & -- & $<.1$ & .0563 & -- & $<.1$ & 9.6 & $<.2$ & $<3$ \\
\hline$<.2$ & 7.1 & 2.7 & .02 & -- & $<.1$ & .0513 & -- & $<.1$ & 9.5 & $<.2$ & $<3$ \\
\hline$<.2$ & 8.2 & 2.9 & $<.02$ & - & $<.1$ & .02 & -- & $<.1$ & 8.5 & .2 & $<3$ \\
\hline$<.2$ & 11.7 & 2.4 & .02 & -- & $<.1$ & .02 & -- & $<.1$ & 9.3 & $<.2$ & 3.1 \\
\hline
\end{tabular}

